Wüst, Wolfgang / Drossbach, Gisela (Hrsg.)

\title{
Umwelt-, Klima- und Konsumgeschichte
}

Fallstudien zu Süddeutschland, Österreich und der Schweiz

Unter Mitarbeit von Lisa Bauereisen und Christoph Gunkel 


\title{
Bibliografische Information der Deutschen Nationalbibliothek
}

Die Deutsche Nationalbibliothek verzeichnet diese Publikation in der Deutschen Nationalbibliografie; detaillierte bibliografische Daten sind im Internet über http://dnb.d-nb.de abrufbar.

Für die Unterstützung der Drucklegung bedanken wir uns bei der Forschungsstiftung Bayerische Geschichte, der Friedrich-Alexander-Universität Erlangen-Nürnberg und der Sektion Franken des Zentralinstituts für Regionenforschung an der FAU.

Umschlagabbildung:

Digitale Bildmontage von Marc Holländer: Dreiklang der Wechselwirkungen, 2018.

Bildnachweise: KHM-Museumsverband Kunsthistorisches Museum Wien GG 1060 Lucas I. van Valckenborch: Sommerlandschaft (Juli oder August)

GG 957 Roelant Savery: Gebirgslandschaft mit Holzfällern GG 3560: Lucas Cranach d. Ä. Hirschjagd des Kurfürsten Friedrich des Weisen

Cover Design: @ Olaf Gloeckler, Atelier Platen, Friedberg

Gedruckt auf alterungsbeständigem, säurefreiem Papier. Druck und Bindung: CPI books $\mathrm{GmbH}$, Leck

\author{
ISBN 978-3-631-77748-0 (Print) · E-ISBN 978-3-631-78135-7 (E-PDF) \\ E-ISBN 978-3-631-78136-4 (EPUB) · E-ISBN 978-3-631-78137-1 (MOBI) \\ DOI 10.3726/b15259 \\ (C) Peter Lang $\mathrm{GmbH}$ \\ Internationaler Verlag der Wissenschaften \\ Berlin 2018 \\ Alle Rechte vorbehalten. \\ Peter Lang - Berlin · Bern · Bruxelles · New York · Oxford · Warszawa - Wien
}

Das Werk einschließlich aller seiner Teile ist urheberrechtlich geschützt. Jede Verwertung außerhalb der engen Grenzen des Urheberrechtsgesetzes ist ohne Zustimmung des Verlages unzulässig und strafbar. Das gilt insbesondere für Vervielfältigungen, Übersetzungen, Mikroverfilmungen und die Einspeicherung und Verarbeitung in elektronischen Systemen.

Diese Publikation wurde begutachtet. 
Stefan Lindl

\section{Klima und Konsum. Gesellschaftliche Konstitution des anthropogen verursachten Klimawandels von 1600 bis zu Svante Arrhenius}

Svante Arrhenius is considered the "father of climate change". The idea of "anthropogenic climate change" allegedly goes back to him. But this statement is not tenable on closer examination of the discourse of climate change. Hardly any position of Svante Arrhenius is genuine. He is in a long tradition of discourse from 1600 until the publication "On the Influence of Carbonic Acid in the Air upon the Temperature of the Ground" (1896). These different positions of discourse are recorded and analyzed in the present study. Svante Arrhenius is reevaluated and classified into the following topics: Glacier, Ice Age, Greenhouse Effect, Atmospheric Carbon Dioxide. The study area is located in the Alps of Savoy, Switzerland and Tyrol.

\section{Diskurs oder „father of climate change“?}

„Klima und Konsum“ - dieser nicht zufällige Zusammenstand zweier Worte impliziert eine korrelierende Verknüpfung, die im 21. Jahrhundert kaum noch in Frage gestellt wird. ${ }^{1}$ Konsum wirkt auf das Klima, wirkt auf den Konsum, wirkt auf das Klima, wirkt auf den Konsum, wirkt auf das Klima... Der anthropogen, aber auch der nicht-anthropogen verursachte Verbrauch von Ressourcen, also deren Konsum durch Lebewesen und auch anorganische Strukturen, übt Einfluss auf deren direkte Umwelt aus. Aber der Konsum greift eben noch viel weiter; er belangt das Klima. ${ }^{2}$ Ressourcen werden genutzt und mit ihrem Verbrauch sowie ihrer Nutzung werden sie in einen anderen energetischen Zustand versetzt. Konsum umfasst eine Vielzahl von Transformationsprozessen. Das sind Vorgänge,

1 Zu den Folgen des Klimawandels vgl. die Seiten des Potsdamer Institutes für Klimafolgenforschung, URL: www.pik-potsdam.de und www.klimafolgenonline.com (letzter Zugriff: 17.9.2018).

2 Anorganischer Konsum impliziert eine weite Definition von Ressourcenverbrauch, die beispielsweise bei der Entstehung von Kristallen und Kalkstein auftritt. Dem Anorganischen wird damit der Status der Aktanten zugeschrieben. 
die nicht wirklich etwas Besonderes darstellen, denn auf ihnen beruht das Leben. Anders formuliert: Wären diese Transformationen von Ressourcen in Energie wie beispielsweise Stoffwechselprozesse nicht möglich, gäbe es kein Leben. Konsum bedeutet Leben. ${ }^{3}$

Die Transformation von Ressourcen in Energie betrifft die endothermen sowie die exothermen Nutzungsarten von Ressourcen. Eine endotherme Nutzung der Ressource Kohlenstoffdioxid findet sich in der Photosynthese. Pflanzen produzieren Sauerstoff und binden gleichzeitig freies Kohlenstoffdioxid. Exotherme Prozesse hingegen finden mitunter in der Verbrennung von Pflanzen statt. Sie verbrauchen Sauerstoff und setzen Kohlenstoffdioxid frei. Es wird dann zum atmosphärischen Kohlenstoffdioxid, das wiederum durch Wetterereignisse als Ressource für endotherme Prozesse verfügbar ist. Ein ausgeglichener Kreislauf könnte dies im Idealfall sein.

Dieses wesentlich idealisierte, vereinfacht und reduziert dargestellte zirkuläre Gleichgewicht von endothermen und exothermen Prozessen bestand bis ungefähr 1800 und wurde dann durch die fossile Revolution aufgebrochen. Waren bis dahin die Territorial- und Staatsökonomien Europas und Nordamerikas überwiegend endotherm-agrarisch strukturiert, so wandelten sich im späten 18. und mit hoher Intensität im 19. Jahrhundert durch die zunehmende Industrialisierung die endothermen Ökonomien in exotherme. Photosynthese, die Grundlage der agrarischen Wirtschaftssysteme, trat in den Hintergrund. Wesentlich höhere und vor allem wetterunabhängige Kapitalerträge als aus endothermen ließen sich aus exothermen Prozessen mit dem Einsatz fossiler Energieträger generieren. Ab der zweiten Hälfte des 19. Jahrhunderts ist die Asymmetrie zugunsten der exothermen Prozesse signifikant festzustellen. ${ }^{4}$ Es wurde seitdem mehr Kohlenstoffdioxid freigesetzt, als es endotherm gebunden werden konnte. In dieser Reduktion der Komplexität endothermer und exothermer Prozesse sind die anderen Speicher des übermäßig freigesetzten Kohlenstoffdioxids nicht aufgeführt, allen voran die Gewässer der Erde, die zunehmend durch das Kohlenstoffdioxid versauern. Mit dem übermäßigen Konsum von Ressourcen achtete die industrialisierte Menschheit seit dem 19. Jahrhundert nicht darauf, die exothermen Prozesse ausreichend auszugleichen und deren Endprodukte transformierend rückzuführen. Die atmosphärische Konzentration von Kohlenstoffdioxid erhöhte sich seitdem durch anthropogene Einflüsse, die auf Konsum von Ressourcen beruhen.

3 Dies gilt, wenn Konsum mit Nutzung und Verbrauch von Ressourcen gleichgesetzt wird.

4 Beispielsweise: Thomas J. Crowley, Causes of Climate Change Over the Past 1000 Years, in: Science 289, 2000, S. 270-277, hier: S. 273. 
Die komplexen Zusammenspiele des Ressourcenkonsums, des Treibhauseffekts, des Klimawandels und der Ressourcenkreisläufe in dieser Form darzustellen, wäre für eine naturwissenschaftliche Studie nicht zulässig. Für die Studien der environmental humanities geht es aber nicht um die Analyse, Aushandeln und Bewerten naturwissenschaftlicher Positionen, sondern beispielsweise um das relationale Verhältnis dieser Positionen zueinander, um ihr diskursives Verhalten untereinander. Die hier vorgelegte Studie möchte die Genese des Diskurses der anthropogenen Einflüsse auf das Klima durch den Konsum nachzeichnen. Seit wann und wie vollzieht sich dieser Diskurs? Aus welchen Diskursen ging er hervor? Wie und an welchen Phänomenen wurde Klimawandel im Allgemeinen festgestellt? Wie formierten sich die Ideen zur Existenz von Klimawandel?

In der Wissenschaftsgeschichte des durch Konsum bedingten Klimawandels herrscht die Meinung vor, der schwedische Physiker, Chemiker sowie Nobelpreisträger Svante Arrhenius habe als erster den anthropogen bedingten Klimawandel im Jahr 1896 formuliert. ${ }^{5}$ Diese Meinung vertritt auch Willy Viehöver mit seiner grundlegenden Studie über die Narrative des globalen Klimawandels. Ihm verdankt der vorliegende Aufsatz seine Existenz. ${ }^{6}$ In der breiten Öffentlichkeit wird Arrhenius sogar als "father of climate change" gehandelt. ${ }^{7}$ Historische Diskursforschung kann eine solche wissenschaftlich gut fundierte Doxa hinterfragen und aufgrund der Analyse langer Linien durch die Jahrhunderte möglicherweise zu anderen Ergebnissen kommen. So lauten die weiteren Fragen in diesem Beitrag: Wie sind Svante Arrhenius' (angebliche primäre, eventuell sogar genuine) Diskurspositionen zum anthropogen bedingten Klimawandel diskursiv einzuordnen und zu bewerten, wenn der sich gesellschaftlich konstituierende Diskurs über

5 Spencer R. Weart, The Discovery of Global Warming, Cambridge/Mass. 2003, S. 6 f.

6 Willy Viehöver, Öffentliche Erzählungen und der Globale Wandel des Klimas, in: Markus Arnold / Gert Dressel / Willy Viehöver (Hg.), Erzählungen im Öffentlichen: Über die Wirkung narrativer Diskurse, Wiesbaden 2012, S. 173-215, hier: S. 187. „Die ersten Erzählungen von einem $\mathrm{CO}_{2}$-bedingten anthropogenen globalen Klimawandel kamen von Arrhenius und Chamberlin (1899) gegen Ende des 19. Jahrhunderts." Mein Dank gilt Willy Viehöver, der mit seinen Studien zum Klimawandel inspirierend wirkte. Seine Forschungen über den frühen Zeitpunkt zum Wissen über den anthropogenen Einfluss auf das Klima, 1896, war ein wegweisender Beitrag für die environmental humanities und die environmental social science. Nur durch eine Arbeit am Text bekommt die naturwissenschaftliche Perspektive ihre im wahrsten Sinne dieses Forschungsbereichs menschliche Dimension. Dazu gehört auch das Vergessen und die Macht der Diskurspositionen, die Meinungen wie die von Svante Arrhenius ausgrenzten und für einige Jahrzehnte in die Marginalität des Forschungsbetriebs drängten.

7 Ian Sample, The father of climate change, in: The Guardian, 30. Juni 2005. 
mehrere Jahrhunderte betrachtet wird? Wie weit lässt sich der Diskurs des Klimawandels in die Vergangenheit zurückverfolgen und an welchen Phänomenen sowie an welchem empirischen Material ist dies überhaupt möglich?

Es bietet sich an, eine chronologische Abfolge weitgehend unberücksichtigt zu lassen. Die vorliegende Studie nimmt sich verschiedene Diskursschichten analytisch vor. Es wird zuerst betrachtet, welche Diskurspositionen Svante Arrhenius bezog und aus welchen Diskursen heraus sie sich formierten. Das heißt, ausgehend von Arrhenius Text werden seine offensichtlichen Verweise auf Autoren untersucht, auf die er sich in Fußnoten bezieht. Zudem wird auch das ihm möglicherweise unbewusste diskursive Feld des 19. Jahrhunderts erfasst und anschließend analysiert. Sodann werden diese Positionen an den neuralgischen Diskurspunkten im 19. Jahrhundert aufgesucht und in Relation zu Arrhenius' Ideen gestellt. Im Anschluss daran springt die Studie zurück ins Mittelalter und versucht ab dem 11. beziehungsweise 12. Jahrhundert einige frühe Spuren des Klimawandeldiskurses zu fixieren, um dann über das 16., 17. und 18. Jahrhundert wieder ins 19. Jahrhundert zurückzukehren. Letztlich wird es möglich, die prominenten Diskurspositionen Svante Arrhenius' neu zu bewerten und ihn in eine lange Tradition einzuordnen. Der Untersuchungsraum, in dem der Klimawandeldiskurs entstanden ist, liegt in den Savoyer, Schweizer und Tiroler Alpen. Von dort und über diesen Raum stammt auch das empirische Material, auf das sich die vorliegende Studie stützt. Die Methode zur Taxierung der Diskurspositionen basiert auf der von mir entwickelten Signifikanten-Interaktionsanalyse. ${ }^{8}$

\section{Svante Arrhenius: Doxa, Episteme, Zuschreibung}

Es gibt zwei Arten des Vaterwerdens, die eine ist materiell, die andere ist diskursiv konstituierend. Beide gehören zusammen. Den Titel „father of climate change“ hat sich Arrhenius nicht selbst gegeben. Es ist eine allzu späte Zuschreibung des 20. und 21. Jahrhunderts. Aber valide Zuschreibungen beruhen auf einer Grundlage. Sie haben deswegen eine materielle Seite. Svante Arrhenius lieferte sie mit einem nahezu gleichzeitig 1896 auf Deutsch und Englisch erschienenen Aufsatz: „Über den Einfluss des atmosphärischen Kohlensäuregehalts auf die Temperatur der Erdoberfläche" und „On the Influence of Carbonic Acid in the Air upon the Temperature of the Ground“. ${ }^{9}$ Die zentralen Diskurspositionen, die Arrhenius

8 Stefan Lindl, Der Umgang mit Gewordenem. Signifikanten-Interaktionsanalyse, Wien 2017.

9 Svante Arrhenius, Über den Einfluss des atmosphärischen Kohlensäurengehalts auf die Temperatur der Erdoberfläche, in: Bihang till Kungl. Svenska Vetenskapsakademiens 
einnimmt, sind präzise formuliert, aber nicht ganz einfach diskursiv zuzuordnen. Die folgende Analyse seines Aufsatzes ruht auf der englischen Version, die im Philosophical Magazine and Journal of Science erschienen ist. Aus Arrhenius' Aufsatz sind folgende Diskurspositionen zu exzerpieren:

1. Die mittlere Temperatur des Bodens ist abhängig von hitzeabsorbierenden Gasen in der Atmosphäre.

"Is the mean temperature of the ground in any way influenced by the presence of heat-absorbing gases in the atmosphere?"10

2. Die Atmosphäre funktioniert wie das Glas eines Treibhauses, es lässt Licht einfallen, aber die Wärmereflektionen des Bodens nicht mehr heraus.

„[Jean Baptiste] Fourier maintained that the atmosphere acts like the glass of a hothouse, because it lets through the light rays of the sun but retains the dark rays from the ground. "11

3. Kohlenstoffdioxid ist für die Absorption der Sonnenstrahlen verantwortlich. „[John] Tyndall held the opinion that the water-vapour has the greatest influence, whilst other authors, for instance [Ernst] Lecher and [Josef Maria] Pernter, are inclined to think that the carbonic acid plays the more important part. ${ }^{\text {"12 }}$

4. Der Konsum von fossilen Energieträgern durch die moderne Industrie führt zu einem signifikanten Anstieg der Kohlenstoffdioxidkonzentration.

"The world's present production of coal reaches in round numbers 500 millions of tons per annum, or 1 ton per $\mathrm{km}^{2}$ of the earth's surface. Transformed into carbonic acid, this quantity would correspond to about a thousandth part of the carbonic acid in the atmosphere. [...] This quantity of carbonic acid, which is supplied to the atmosphere chiefly by modern industry, may be regarded as completely compensating the quantity of carbonic acid that is consumed in the formation of limestone (or other mineral carbonates) by the weathering of decomposition of silicates. ${ }^{\text {"13 }}$

5. Der Eiszeitdiskurs des 19. Jahrhunderts und die Absenz einer schlüssigen Erklärung brachte Arrhenius zur Hypothese, dass eine Absenkung des atmosphärischen Kohlenstoffdioxids zur globalen Abkühlung führte.

Handlingar 22/1, 1896, S. 1-102; Ders., On the Influence of Carbonic Acid in the Air upon the Temperature of the Ground, in: Philosophical Magazine and Journal of Science, Series 5, 41, 1896, S. 237-276.

10 Ebd., S. 237.

11 Ebd.

12 Ebd., S. 239.

13 Ebd., S. 270. 
"In the Physical Society of Stockholm there have been occasionally very lively discussions on the probable causes of the Ice Age, in my opinion, led to the conclusion that there exists as yet no satisfactory hypothesis that could explain how the climatic conditions for an ice age could be realized in so short a time as that which has elapsed from the days of the glacial epoch. ${ }^{14}$

6. Die Konzentration von Kohlenstoffdioxid in der Atmosphäre hat gravierende Auswirkungen auf die Temperatur der Erde. Die 2,5-3fache Menge atmosphärisches $\mathrm{CO}_{2}$ bedeutet einen Temperaturanstieg von $8^{\circ}-9^{\circ} \mathrm{C}$ in der Arktis.

„[...] Conversations with my friend and colleague Professor [Arvid Gustaf] Högbom, [...] led me to make a preliminary estimate of the probable effect of a variation of the atmospheric carbonic acid on the temperature of the earth. As this estimation led to the belief that one might in this way probably find an explanation for temperature variations of $5^{\circ}-10^{\circ} \mathrm{C}$. ${ }^{\prime 15}$

„A simple calculation shows that the temperature in the arctic regions would rise about $8^{\circ}$ to $9^{\circ} \mathrm{C}$., if the carbonic acid increased to 2.5 or 3 times its present value. In order to get the temperature of the ice age between the 40th and 50th parallels, the carbonic acid in the air should sink to $0.62-0.65$ of its present value (lowering of temperature $4^{\circ}-5^{\circ} \mathrm{C}$.). ${ }^{\text {"16 }}$

7. Ozeane als Speicher von Kohlenstoff.

"From the determination of the amounts of dissolved substances, especially carbonates, in a number of rivers in different countries and climates, and of the quantity of water flowing in these rivers and of the quantity of water flowing countries and climates, and of the quantity of water flowing in theses rivers and of their drainage-surface compared with the land-surface of the globe, it is estimated that the quantities of dissolved carbonates that are supplied to the ocean in the course of a year reach at most the bulk of $3 \mathrm{~km}^{3}{ }^{\text {" } 17}$

Einiges an diesen Diskurspositionen, die Arrhenius niederschrieb, ist bemerkenswert. Zuerst scheint der schwedische Chemiker damit einen Großteil des Wissens über den Klimawandel abgedeckt zu haben. Im Wesentlichen entspricht sein Wissen dem des 21. Jahrhunderts. Eine genauere Einordnung Arrhenius' Positionen in die verschiedenen Diskurse des 19. Jahrhunderts lassen allerdings seine primärgenuine Stellung, die ihm heute zugeschrieben wird, in einem differenzierteren Licht erscheinen:

Die Positionen 1-3 übernahm Arrhenius nach eigenen Angaben von Jean Baptiste Fourier, John Tyndall, Josef Maria Pernter, Ernst Lecher. So findet sich die Diskursposition 1 im Mémoire von Jean Baptiste Fourier von 1827: „Il est difficile de connaître jusqu’à quel point de l'atmosphère l'influe sur la température

14 Ebd., S. 267.

15 Ebd.

16 Ebd., S. 268.

17 Ebd., S. 270. 
moyenne du globe. ${ }^{18}$ Dass die Atmosphäre die mittlere Temperatur beeinflusst, daran zweifelte Fourier nicht. So kam Fourier zu dem Ergebnis: „c'est ainsi que la temperature est augmentée par l'interposition de l'atmosphère, parce que la chaleur trouve moins d’obstacle pour pénétrer l'air, étant à l'état de lumière, quelle n'en trouve pour repasser dans l'air lorsquelle est convertie en chaleur obscure. “" („So wird die Temperatur durch das Dazwischentreten/die Zwischenschaltung der Atmosphäre erhöht, weil die Hitze in Lichtform weniger Hindernis findet, um in die Luft einzudringen, als wenn sie in infrarote Strahlung verwandelt ist.") „La chaleur, arrivant à l'état de lumière jusqu’à la terre solide, perdrait tout-à-coup et presque entièrement la faculté qu'elle avait de traverser les solides diaphanes; elle saccumulerait dans les couches inférieures de l'atmosphère, qui acquerraient ainsi des températures élevées. “20 („Die Hitze, die im Licht-Zustand die feste Erde erreicht, würde plötzlich und fast vollständig die Fähigkeit verlieren, die durchscheinenden Festkörper zu durchqueren; es würde sich in den unteren Schichten der Atmosphäre anreichern, die somit hohe Temperaturen erreichen würden.") Ebenso enthält John Tyndalls Text diese Position: „The waves of heat speed from our earth through our atmosphere towards space. Theses waves dash in their passage against the atoms of oxygen and nitrogen, and against the molecules of aqueous vapour. " ${ }^{21}$ Tyndall übernimmt die Position Fouriers und passt sie an sein Wissen an, indem er sie um die Zusammensetzung der Atmosphäre aus Sauerstoff, Stickstoff und Wasserdampf bereicherte. Jedoch konnte er nicht auf das Kohlenstoffdioxid verweisen. Dessen Virulenz als hitzeabsorbierendes Gas wurde erst 1881 in den Diskurs eingeführt (vgl. Ernst Lecher, 1881).

Die Diskursposition 2 von Arrhenius enthält die Metapher des Treibhauses bezugnehmend auf Jean Baptiste Fourier. Fouriers Text weist sie jedoch nicht auf. Aber er beschrieb die Funktionsweise eines Treibhauses durch seine Versuchsanordnung. Mit einem Messinstrument, das Horace-Bénédict de Saussure bereits im 18. Jahrhundert entwickelt hatte, wies er auf das hin, was Arrhenius prägnant

18 Jean Baptiste Fourier, Mémoire sur les températures du globe terrestre et des espaces planétaires, in: Mémoires de l'Académie royale des sciences de l'Institut de France 7, 1827, S. 569-604, hier: S. 584.

19 Ebd., S. 587.

20 Ebd., S. 585.

21 John Tyndall, On Radiation through the Earth's Atmosphere, in: The London, Edinburgh, and Dublin Philosophical Magazine and Journal of Science 4, 1863, S. 200-206, hier: S. 202. 
den „Treibhauseffekt" nannte..$^{22}$ Es handelte sich um ein Gefäß, das mit Glasplättchen abgedeckt wurde. Zur Isolierung wurde diese Schachtel mit geschwärztem Kork ausgekleidet. In verschiedenen Höhen wurden Thermometer eingesetzt. Je weiter sie von den Glasplättchen entfernt waren, desto geringer, je näher sie an den Glasplättchen positioniert waren, desto höher war ihre Temperatur. ${ }^{23}$ „Elle consiste à exposer aux rayons du soleil un vase couvert d'une ou de plusieurs lames de verre bien transparent. ${ }^{\text {" } 4}$

Zur Diskursposition 3: Der Nachweis für die Bedeutung des Kohlenstoffdioxids als Hauptverursacher des Treibhauseffekts und der Absorption der Sonnenstrahlen wurde allerdings nicht von dem Autorenduo Pernter und Lecher erbracht, wie Arrhenius intendiert. In ihrem gemeinsamen Aufsatz von 1881 kamen Pernter und Lecher zu dem Schluss: „Nach dem Gesagten ist klar, dass einstweilen eine definitive Lösung der Frage nach dem eigentlichen Absorbers in unserer Atmosphäre noch nicht gegeben werden kann, umso mehr, da der Kohlensäuregehalt der Luft und auch all' die organischen pflanzlichen und thierischen Organismen, welche in der Atmosphäre schweben, bei Beurteilung dieses Gegenstandes von Einfluss sein werden. . $^{\text {" }}$ Wenig später, aber doch noch im selben Jahr 1881, veröffentlichte Ernst Lecher ohne Josef Maria Pernter einen weiteren Aufsatz, in dem Lecher die tragende Rolle des Kohlenstoffdioxid nachweisen konnte. ${ }^{26}$ Lecher fasste seine Ergebnisse folgendermaßen zusammen: „Es ist also jene Menge $\mathrm{CO}_{2}$, welche nach dem bisherigen meteorologischen Wissen sich über uns befindet, mehr als ausreichend, die atmosphärische Absorption der Sonnenstrahlung zu erklären. “27

Die Position 4 betrifft die Industrie mit ihrem Kohlenstoffdioxidausstoß durch Kohleverbrennung. In dieser Diskursposition wird der anthropogene Einfluss auf den Treibhauseffekt und auf den daraus resultierenden Klimawandel deutlich und signifikant betont. Allerdings stammt sie, bei genauer Betrachtung des Texts, von dem Geologen Arvid Gustaf Högbom, dem Freund von Svante Arrhenius. Korrekt

22 René Sigrist, Le capteur solaire de Horace-Bénédict de Saussure. Genèse d’une science empirique, Genève 1993.

23 Fourier, Mémoire (wie Anm. 18), S. 585.

24 Ebd.

25 Ernst Lecher / Joseph Pernter, Über die Absorption dunkler Wärmestrahlen in Gasen und Dämpfen, in: Sitzungsberichte der Mathematisch-Naturwissenschaftlichen Classe der Kaiserlichen Akademie der Wissenschaften: Mathematik, Physik, Chemie, Mechanik, Meteorologie und Astronomie. Abteilung II, 82, 1981, S. 180-201, hier: S. 199 f.

26 Ernst Lecher, Über die Absorption der Sonnenstrahlung durch die Kohlensäure unserer Atmosphäre, in: Annalen der Physik 248, 1881, 3, S. 466-473.

27 Ebd., S. 471. 
werden diese Abschnitte auch als Zitate von Högbom gekennzeichnet. Allerdings vernachlässigt die Literatur über Arrhenius den Verweis auf Gustaf Arvid Högbom. So auch Willy Viehöver: „Svante Arrhenius hat nicht nur die Theorie des Treibhauseffektes fortentwickelt, er formulierte auch einen Plot, der die künftige Erwärmung des Planeten als durch industrielle Verbrennung von Kohle verursacht beschreibt.“ Danach zitiert Viehöver Arrhenius: „Man hat sogar berechnet, dass die jetzige Industrie soviel Steinkohlen verbraucht, dass sie in 1.000 Jahren, also geologisch genommen einer äusserst kurzen Frist, ebensoviel Kohlensäure produzieren würde, wie der ganze Kohlensäureinhalt der Atmosphäre. Zwar spielt die teils physikalische teils chemische Absorption der Kohlensäure im Meerwasser hier eine regulierende Rolle, aber es ist doch ersichtlich, dass der Kohlensäuregehalt der Atmosphäre in recht kurzen Zeiten eine bedeutende Schwankung erfahren kann, und wahrscheinlich sogar erfahren muss. ${ }^{28}$ In der englischen Ausgabe von Arrhenius' Aufsatz wird die von Viehöver zitierte Passage folgendermaßen eingeleitet: „Is it probable that such great variations in the quantity of carbonic acid as our theory requires have occurred in relatively short geological times? The answer to this question is given by Prof. Högbom. As his memoir to this question may not be accessible to most readers of these pages, I have summed up and translated his utterances which are of most importance to our subject. ${ }^{\text {29 } 9}$ Arrhenius übersetzt einen Aufsatz Högboms von 1894 mit dem Titel „Om sannolikheten för sekulära förändringar i atmosfärens kolsyrehalt“ („Untersuchungen über den Kohlensäuregehalt der Atmosphäre"). ${ }^{30}$ Högbom ist mit seinem Hinweis auf die Industrie nicht der Erfinder des anthropogenen Einflusses. Er liegt diskursiv zeitlich vor der Schrift Högboms schon bei Fourier vor. Högbom fomulierte: „This quantity of carbonic acid, which is supplied to the atmosphere chiefly by modern industry [...]. “" Högbom stellte den Zusammenhang von „modern industry“ und „carbonic acid“ heraus, im Gegensatz zur „l'industrie“ bei Fourier, der die Relation Industrie und ihren Einfluss auf die Temperatur erfasste: „Les effets de l'industrie humaine

28 Viehöver, Öffentliche Erzählungen (wie Anm. 6), S. 187, nimmt Bezug auf Arrhenius, Über den Einfluss (wie Anm. 19), S. 66.

29 Arrhenius, On the Influence (wie Anm. 9), S. 269.

30 Arvid Gustaf Högbom, Om sannolikheten för sekulära förändringar i atmosfärens kolsyrehalt, in: Svensk Kemisk Tidskrift 6, 1894, S. 169-176. Eine kurze Zusammenfassung des Aufsatzes erschien im Chemischen Centralblatt 1, 1897, S. 452. Im Diskursfeld um Högbom steht auch der bayerische Landtechniker Heinrich Puchner, Untersuchungen über den Kohlensäuregehalt der Atmosphäre, in: Forschungen auf dem Gebiete der Agrikulturphysik 15, 1892, S. 296.

31 Arrhenius, On the Influence (wie Anm. 9), S. 270. 
[...] modifient les températures dans chaque climat. ${ }^{\text {"32 }}$ Bei Högbom fand also eine Anpassung der Diskurspositionen des 19. Jahrhunderts statt, die auf seine Kalksteinforschung ausgerichtet ist. Diese Differenz negiert aber keineswegs die entscheidende Übereinstimmung: Die anthropogenen Einflüsse der menschlichen Geschäftigkeit auf die Umwelt. Genau genommen fehlt in Högboms Text der Einfluss auf das Klima, den Fourier 1827 bereits formuliert hatte. Erst aus der Kombinatorik dieses von Högboms beschriebenen Effekts mit den Ergebnissen der Studie von Ernst Lecher über den Treibhauseffekt durch das Kohlenstoffdioxid, konnte Arrhenius Fouriers „'lindustrie“ und Högboms „industry“ miteinander in einen argumentativen Einklang bringen. Somit behält Willy Viehöver recht: Ohne die Kombinatorik der einzelnen Diskursbestandteile durch Arrhenius, wäre die Position des anthropogen bedingten Klimawandels durch den Treibhauseffekt nicht zu dem Zeitpunkt 1896 möglich gewesen. Arrhenius entwickelte den Diskurs also kombinatorisch weiter. Unzweifelhaft ist jedoch auch, dass seit 1827 und wahrscheinlich schon davor ein Diskursbestandteil existierte, der die exothermen Geschäfte der Menschen und das Klima miteinander verknüpften.

Die Diskursposition 5 erläutert, aus welchem Diskurs Svante Arrhenius auf den anthropogen verursachten Klimawandel gestoßen ist. Es war der Eiszeit- und Gletscherdiskurs, der nicht nur in Schweden stark ausgeprägt, sondern während des gesamten 19. Jahrhunderts überall in Europa geführt wurde. Die Eiszeit(en) und die Ausdehnung der Gletscher zu einer vergangenen Zeit, ermöglichten es allein, über Klimawandel nachzudenken. Der Eiszeitdiskurs war in gewisser Weise wohl auch beängstigend. Sollte es möglich sein, dass die zivilisatorischen Errungenschaften im flachen Land, weit entfernt von gegenwärtigen Gletschern, in latenter Gefahr schwebten, von Eis bedroht zu sein, ähnlich inaktiver Vulkane, mit deren Ausbrüchen zu rechnen sei? Der lebhaft geführte Diskurs in der Physical Society of Stockholm über die Eiszeit sowie die Forschungsergebnisse von Fourier, Tyndall, Pernter, Lecher und Högbom führten zu den Diskurspositionen Arrhenius': Den Treibhauseffekt, der durch das atmosphärische Kohlenstoffdioxid bedingt wird, wendete er als Erklärung für die Eiszeit an. Der Zusammenhang von Gletscherausdehnung und Temperatur bestimmt durch die Atmosphäre findet sich jedoch auch früher in Louis Agassiz' „Études sur les glaciers" von 1840. ${ }^{33}$ Weil das Thema der Ausdehnung der Gletscher und ihre extreme Form,

32 Fourier, Mémoire (wie Anm. 18), S. 584.

33 Vgl. Der Gletscherdiskurs im 19. Jahrhundert in diesem Aufsatz. Zum Zusammenhang von Gletscherausdehnung und Atmosphäre: Louis Agassiz, Études sur les glaciers, Neuchatel 1840, S. 201. 
die Eiszeit, der bestimmende Diskurs zu sein scheint, der Klimawandel überhaupt erst denkbar gemacht hatte, wird seine Entwicklung gesondert und ausführlich betrachtet. Alpengletscher waren der Zugang zum Klimawandel. Die Alpen boten sich zudem an, in „Himmelsnähe“ Experimente durchzuführen. Es ist deswegen nicht unerheblich auf Horace de Saussure und seine Arbeit am Ende des 18. Jahrhunderts zu verweisen, der einerseits Gletscher untersuchte, andererseits mit seinen Messinstrumenten die Grundlagen für die Erforschung des Treibhauseffekts vorbereitete. ${ }^{34}$ Darin liegt auch die strukturelle Verbindung von de Saussure und Tyndall: John Tyndall war der Horace de Saussure des 19. Jahrhunderts - Naturwissenschaftler und Bergsteiger zugleich.

Mit Diskursposition 6 fügt Arrhenius dem Klimawandeldiskurs eine Neuheit hinzu, eine simulierend-modellierende Rechnung, welche Temperaturänderung sich ergibt, wenn sich die Kohlenstoffdioxidkonzentrationen in der Atmosphäre verändern. Erhöht sie sich, erhöht sich auch die mittlere Erdtemperatur. Sinkt die Konzentration, dann wird es kälter und das bislang unerklärliche Phänomen einer Eiszeit sowie das Anwachsen von Gletschern bekommt eine evidente Erklärung. Diese Diskursposition scheint die einzige genuine Leistung von Svante Arrhenius in seinem Aufsatz zu sein, weil er auf keinen Kollegen in Fußnoten verweist. Wird jedoch die auf Italienisch erschienene Publikation von Luigi de Marchi „Le cause dell' era glaciale“ von 1895 herangezogen, so schwindet in gewissem Maße auch diese Beurteilung von Arrhenius' Diskursposition. De Marchi berichtet von der Diskussion des Einflusses der Atmosphäre auf die Eiszeit in Italien und diskutiert den Zusammenhang von Atmosphäre Termperatur und die teils abenteuerlichen Ansichten und Schlussfolgerungen seiner Kollegen. So schreibt de Marchi: „Esamina e trova del pari non accettabile l'opinione che attribuirebbe l'era glaciale ad una temperatura più elevata dell' atmosfera e ad una conseguente maggior evaporazi-

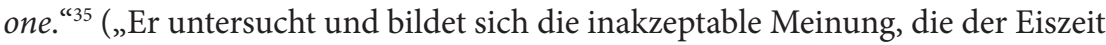
eine höhere Temperatur der Atmosphäre und einer daraus folgenden stärkeren Verdunstung zuschreiben würde.“) De Marchis’ Monographie enthält allein 126

34 Auf Horace de Saussure wird später noch ausführlicher eingegangen. Auf seinen Expeditionen auf die Alpengipfel begleitete ihn eine Schar von Lastenträgern, die beispielsweise auf den Montblanc in mehreren Kisten die Instrumente für die Experimente de Saussures trugen. Horace de Saussure, Kurzer Bericht von einer Reise auf den Gipfel des Montblanc, im August 1787, Strasburg 1788, S. 5: „Ich begab mich den ersten August in Begleitung eines Bedienten und 18 Führer die meine physikalischen Werkzeuge und alles nöthige Geräte trugen, auf den Weg. "Dort findet sich auch ein Namensverzeichnis der Teilnehmer dieser Expedition.

35 Luigi de Marchi, Le cause dell'era glaciale, Pavia 1895, S. VI. 
Treffer für das Wort „atmosfera“. Der Meteorologe de Marchi berechnet und diskutiert nichts anderes als den Einfluss der Atmosphäre auf die Eiszeit. Arrhenius verweist auch auf ihn, aber nicht an der Stelle, die de Marchi gebührte. Auf Seite 267 erwähnt er, wie er auf die Atmosphärentheorie kam. Dort hätte sich auch ein Verweis auf de Marchi gut gemacht. Aber erst auf Seite 273 verweist er auf den Italiener. Dort geht es um die Auflistung bestehender Eiszeittheorien, die alle nach de Marchi abzulehnen seien. Allerdings sieht de Marchis Erklärung für die Eiszeit die Transparenz der Atmosphäre an. Wasserdampf sei dafür verantwortlich - nicht wie bei Arrhenius das Kohlenstoffdioxid. De Marchi kannte folglich die Arbeit von Ernst Lecher nicht, sondern argumentiert noch mit den Diskurspositionen John Tyndalls. Aber trotzdem sieht de Marchi die Atmosphäre und den Treibhauseffekt als Grund für den eiszeitlichen Klimawandel an und erklärt sie auch damit. Arrhenius passte de Marchis Diskursposition mit der von Ernst Lecher an. Darin liegt die Stärke und das Verdienst Svante Arrhenius'. Er kombinierte, das ist sein Diskurspotential. Er führte die Positionen von Agassiz, Tyndall, de Marchi, Lecher, Fourier und Högbom zusammen.

Wiederum von Högbom stammt Diskursposition 7. Sie simuliert, was durch einen anthropogen bewirkten Anstieg der Kohlenstoffdioxidkonzentration in den Ozeanen passiert: Sie dienen als Pufferspeicher für die nicht gelösten Karbonate. Implizit steckt darin auch die Übersäuerung der Gewässer, die bei weiterer und intensivierter Emission von $\mathrm{CO}_{2}$ sich vollzieht.

Dreh- und Angelpunkt der anthropogen bedingten Treibhaustheorie ist die Eiszeit, das heißt das Anwachsen der Gletscher. Sie war für die Naturforscher der offensichtlichste Klimawandel im 19. Jahrhundert, der als wissenschaftlich belegtes Phänomen einer plausiblen naturwissenschaftlichen Erklärung harrte. Die Eiszeit als Phänomen des Klimawandels scheint der entscheidende diskursive Punkt zu sein, der diese für den anthropogen verursachten Klimawandel schwerwiegenden Positionen ermöglichte. Welche Rolle spielte also die Eiszeit mit ihren sich ausdehnenden Gletschern?

\section{Klimawandel, Eiszeittheorien und die Frage nach der Erdtemperatur im 19. Jahrhundert}

Arrhenius' und Högboms Überlegungen befinden sich - wie angedeutet - in einem langen Diskurs des 19. Jahrhunderts über den Klimawandel am Beispiel der Eiszeit. Wie sich zeigen wird, waren verschiedene Gletscherausdehnungen auch im Zusammenhang mit Klimawandel schon lange bekannt. Eine neue Qualität erreichte dieser Diskurs durch eine Erklärung, wenn auch keine grundlegende, warum sie sich ausdehnten und wieder abschmolzen. Für diese Erklärung war 
der Mannheimer Karl Friedrich Schimper nicht unbedeutend. 1837 verfasste er anlässlich des Geburtstags von Galileo Galilei in Neuchâtel ein Gedicht mit dem Titel „Die Eiszeit“ ${ }^{36}$ Darin beschrieb Schimper Warm- und Kaltzeiten und damit implizit Klimawandel. Die Form des Gedichts scheint außergewöhnlich für eine wissenschaftliche Theorie. Deswegen, um kein Missverständnis aufkommen zu

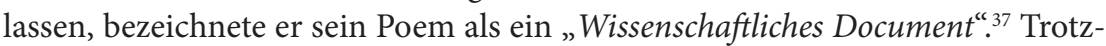
dem trägt es, der lyrischen Form geschuldet, vor allem narrativ-fiktionale Züge: Ein Eisbär tritt als Hauptprotagonist auf, der nun - lange nach der Eiszeit - im Norden wohne, weil es ihm in Mitteleuropa zu warm geworden sei.

„Mehr als das Reich rings, fesseltest du den Sinn,

Eisbär des Nordpols! Führst mich in Gegenden,

Wo winterfroh du noch im Treibeis

Wohnst und behaglich dich übst im Fischfang.

Wohnst hingedrängt dort lange bereits, doch einst

War deine Heimath näher bei uns! Es war

Vielleicht das Urland deiner Schöpfung,

Winterbedeckt noch, das Herz Europas.

Wohl war zuvor mild, milder als jetzt, die Welt:

Weithin im Urwald hallte Gebrüll des Rinds,

Mammuthe grasten still, in Mooren

Wälzten sich lüsterne Pachydermen."

In diesen Versen erfasst Schimper zwei Warmzeiten und eine Eiszeit: Die aktuelle Warmzeit, die den Eisbären an den Nordpol vertrieben hat, eine Kaltzeit, in der das „Herz Europas" seine Heimat gewesen sein soll und eine weitere Warmzeit, in der Mitteleuropa Urwald beheimatete, aber auch Rinder und Mammute sowie Pachydermen (Dickhäuter). Alle seien sie von einer Kaltzeit hinweggerafft worden, wie in den nächsten Versen beschrieben wird:

„Ureis von damals, als die Gewalt des Frosts

Berghoch verschüttet selbst den Süden,

Ebnen verhüllt so Gebirg als Meere!

36 Karl Friedrich Schimper, Die Eiszeit, in: Ders., Gedichte, Erlangen 1840, S. 301-304.

37 Vgl. ebd., S. 301. Die Zuschreibung „Wissenschaftliches Document“ erklärt sich aus dem Plagiatsstreit Schimpers mit Louis Agassiz, der angeblich - so der Vorwurf Schimpers - seine wissenschaftliche Eiszeittheorie aus Schimpers Gedicht unlauter übernommen habe. 
Wie stürzte Schneesturm, welche geraume Zeit,

Endlos herab! Wie, reiche Natur, begrubst

Du lebenscheu dich, öd und trostlos!

Aber es ging ja zuletzt vorüber!"

Doch auch diese Kaltzeit endete und wurde wiederum von einer Warmzeit abgelöst, die schließlich den Eisbären in die kälteren Zonen nach Norden abdrängte. Bemerkenswert ist die Vorstellung Schimpers, warum es diese Warmzeit gab:

„Als nach dem Ausbruch dieser Gewaltigen

Hinsank des Frosts Reich, lebengeschwellte Natur

Der aus sich selbst erwärmten Erde“. ${ }^{38}$

Die Erde, so schrieb er, habe sich aus sich selbst erwärmt. Wie sie das getan haben solle, berichtet Schimper nicht. Aber es wird deutlich, dass die Eiszeiten und Warmzeiten abhängig von der inneren Temperatur der Erde seien. Eine Angabe darüber, woher er diese thermodynamische Überlegung habe, bleibt er schuldig. Allerdings steht seine Erklärung für die Warmzeit im diskursiven Zusammenhang mit Gustav Bischofs „Die Wärmelehre des Innern unsers Erdkörpers, ein Inbegriff aller mit der Wärme in Beziehung stehender Erscheinungen in und auf der Erde“, die ebenfalls 1837 erschien. ${ }^{39}$ Auffällig deckt sich Schimpers Beschreibung der Warmzeit, mit Gustav Bischofs Beantwortung seiner forschungsleitenden Frage, ob die Erde langsam erkalte oder die Sonnenstrahlung die Erkaltung der Erdoberfläche verhindere. Diese Diskussion findet sich im vierten Abschnitt (Kapitel 27) seiner Wärmelehre. ${ }^{40}$ Allerdings weist Bischof daraufhin, dass die Wärme des Erdinnern nicht verantwortlich für die Temperatur an der Erdoberfläche sei. ${ }^{41}$ Davon weicht Schimper ab. Er folgt eher den Studien von Horace de Saussure aus dem 18. Jahrhundert, der Gegenteiliges vermutete. ${ }^{42}$ Schimper griff also auf das

38 Schimper, Gedichte (wie Anm. 36), S. 303.

39 Gustav Bischof, Die Wärmelehre des Innern unseres Erdkörpers, ein Inbegriff aller mit der Wärme in Beziehung stehender Erscheinungen in und auf der Erde, Leipzig 1837.

40 Ebd., S. 339-341.

41 Über das Abschmelzen der Gletscher an ihren Böden: „Die normale Temperatur-Abnahme von dem Innern der Erde bis zur Oberfläche wird also durch eine Schnee-Bedeckung an einer Stelle nicht dauernd gestört, wo die mittlere Bodenwärme Null ist, und es ist daher nicht denkbar, wie von dem Innern bis dahin jemals ein stärkerer Zug von Wärme statt finden sollte, welcher vermögend wäre, Schnee zu Schmelzen."Vgl. Bischof, Wärmelehre (wie Anm. 39), S. 102. Dazu auch S. 168 f.: „Über den Einfluss des Klimas auf die Temperatur des Erdinnern."

42 Vgl. „L'une de ces causes est la chaleur intérieure de la Terre, qui fait fondre les neiges \& les glaces, même pendant les froids les plus rigoureux, lorsque leur épaisseur est assez grande 
Wissen und Deuten des 18. Jahrhunderts zurück, obgleich Bischof eine weiterentwickelte Thermodynamik veröffentlicht hatte und sich viele diskursive Ähnlichkeiten auftun.

Louis Agassiz, der von Schimper lange Zeit verehrte Freund aus gemeinsamen Studientagen an der Universität Heidelberg, erarbeitete möglicherweise mit den Positionen aus dem Gedicht seine wissenschaftlich fundierte Eiszeittheorie in den „Études sur les glaciers“, die er drei Jahre nach Schimper 1840 publizierte. ${ }^{43}$ Ein Verweis auf Schimpers Gedicht fehlt in Agassiz' ausführlichem Forschungsstand, der nur wissenschaftliche Aufsätze und Monographien erwähnt. Der Freundschaft der beiden war dies nicht sonderlich zuträglich. Sie endete in einem vor allem in der Augsburger Allgemeine Zeitung ausgetragenen Streit, deren europäische Tragweite nur erstaunlich zu nennen ist. ${ }^{44}$ Allein diese Debatte über die Plagiatsvorwürfe bezüglich der Eiszeittheorie macht deutlich, wie wichtig dieses Thema in der gelehrten Öffentlichkeit des 19. Jahrhunderts war, wie gesellschaftlich weitreichend in der ersten Hälfte des 19. Jahrhunderts Klimawandel am Beispiel der Eiszeit diskutiert wurde. Die Streitigkeiten zwischen Schimper und Agassiz über die eigentliche Urheberschaft der Eiszeittheorie sind hier jedoch nebensächlich. Interessant sind deren Argumentationen, in der sich erhebliche Divergenzen auftun. Während Schimper in seinem Gedicht auf die Erderwärmung von innen den Grund für den Klimawandel sieht - Horace de Saussure folgend -, deutet Louis Agassiz das Phänomen der Kalt- und Warmzeiten völlig anders: „La température est l'agent essentiel de la formation des glaciers, de leur extension et de leurs

pour préserver du froid extérieur les fonds sur lesquels elles reposent. " Horace-Bénédict de Saussure, Voyages dans les Alpes, précédés d'un Essai sur l'histoire naturelle des environs de Genève, 4 Bde., Neuchatel 1797, hier: Bd. 1, S. 451, $\$ 532$.

43 Agassiz, Études (wie Anm. 33). In den zeitlichen Umkreis von Agassiz und Schimper gehört auch Franz Joseph Hugi und seine Veröffentlichungen über Gletscher und Findlinge. Franz Joseph Hugi, Über das Wesen der Gletscher und Winterreise in das Eismeer, Stuttgart 1842; Ders., Die Gletscher und die erratischen Blöcke, Solothurn 1843.

$44 \mathrm{Zu}$ dem öffentlich ausgetragenen Streit zwischen Agassiz und Schimper: Anonym, Die Eiszeit und ihre Entdecker, Allgemeine Zeitung 92, 2. April 1842, Beilage, S. 729-731; und: Anonym, Die Eiszeit und ihre Entdecker (Beschluß), in: Allgemeine Zeitung 93, 3. April 1842, Beilage, S. 737-740, besonders die Einschätzung S. 739; dazu auch der Kanzler der Republik Neuenburg: Favarger, Offener Brief, ohne Titel, in: Allgemeine Zeitung 112, 22. April 1842, Beilage, S. 894; Karl Schimper, Replik auf den Brief von Favarger, in: Allgemeine Zeitung 126, 6. Mai 1842, Beilage, S. 1005; auch: Louis Agassiz, Erwiderung auf Dr. Karl Schimpers Angriffe von L. Agassiz, in: Allgemeine Zeitung 295, 22.10.1842, Beilage, S. 2-4. 
mouvemens. L'on conçoit dès lors combien il importerait de connaître exactement toutes les causes qui peuvent modifier les variations auxquels létat de l'atmosphère et $d u$ sol de nos Alpes est soumis. “45 („Die Temperatur ist der wichtigste Faktor für die Gestalt der Gletscher, ihrer Ausdehnung und ihrer Bewegungen. Es ist leicht einzusehen, dass es wichtig wäre, alle Ursachen genau zu kennen, die die Veränderungen bewirken können, welche dem Zustand der Atmosphäre und des Bodens unserer Alpen unterworfen sind.")

Für die gletschergestaltende Temperatur macht Agassiz Atmosphäre und Boden verantwortlich, also die innere Temperatur der Erde sowie die äußere. ${ }^{46}$ Agassiz erweiterte folglich die Schimper'sche thermodynamische Kausalität des Erdinnern um die Atmosphäre. Die Auswirkungen der Atmosphäre auf die Temperatur der Erde war eine Entdeckung, die dreizehn Jahre zuvor, 1827, von Jean Baptiste Joseph Fourier publiziert worden war. Es handelte sich dabei um das bereits erwähnte „Mémoire sur les températures du globe terrestre et des Espaces planétaires“ ${ }^{47}$ Fourier ging es um die Frage nach der Erwärmung der Erde, worauf sie beruhe. Seine Erklärung brachte die Atmosphäre ins Spiel: „c'est ainsi que la temperature est augmentée par l'interposition de l'atmosphère, parce que la chaleur trouve moins d'obstacle pour pénétrer l'air, étant à l'état de lumière, quelle n'en trouve pour repasser dans l'air lorsquelle est convertie en chaleur obscure." ${ }^{8}$ („So erhöht sich die Temperatur durch das Dazwischentreten der Atmosphäre, weil die Wärme in Lichtform weniger Hindernis erfährt, um in die Luft einzudringen, als wenn sie in infrarote Strahlung verwandelt ist.") Diese Position entspricht dem, was heute als natürlicher Treibhauseffekt bezeichnet wird. Fouriers Atmosphäre-Erklärung für die Erwärmung wird von Louis Agassiz als gegeben vorausgesetzt. Es findet sich keine Fußnote oder ein Verweis auf die Studie von Fourier. Auch zu diesem thermodynamischen Diskurs gehörig ist das kurz vor Agassiz' Publikation erschienene „Mémoire sur la chaleur solaire, sur les pouvoirs rayonnants et absorbants de l'air atmosphérique et sur la température de l'espace" von Claude Servais Mathias Pouillet. ${ }^{49}$ Pouillet lässt sich als Mittler zwischen Fourier und John Tyndall in der Atmosphärenforschung identifizieren. In Agassiz' Text über die Gletscher fehlt er. John Tyndall berief sich explizit auf

45 Vgl. Agassiz, Études (wie Anm. 33), S. 201.

46 Pouillet nennt diese Temperaturarten: enceinte und enveloppe. Vgl. Claude Servais Mathias Pouillet, Mémoire sur la chaleur solaire, sur les pouvoirs rayonnants et absorbants de l'air atmosphérique et sur la température de l'espace, Paris 1838, S. 21.

47 Fourier, Mémoire (wie Anm. 18).

48 Ebd., S. 587.

49 Pouillet, Mémoire (wie Anm. 46). 
Pouillet. Verweise auf die Arbeiten von Agassiz und Schimper als Kombinierer der Gletscherforschung und der Thermodynamik fehlen bei Tyndall. Deswegen gilt Tyndalls Text heute in der Wissenschaftsgeschichte der Klimaforschung als die wichtigste Verknüpfungsstudie beider Diskurse. Er wies auch explizit auf seine Leistung hin: "The researches on glaciers which I have had the honour of submitting from time to time to the notice of the Royal Society, directed my attention in a special manner to the observations and speculations of De Saussure, Fourier, M. Pouillet, and Mr. Hopkins, on the transmission of solar and terrestrial heat through the earth's atmosphere. " ${ }^{50}$ Gletscher einerseits, die Arbeiten zur Thermodynamik, Atmosphäre und Treibhauseffekt von de Saussure, Fourier, Pouillet und Hopkins andererseits, ermöglichten ihm die Verknüpfung. Agassiz, der einundzwanzig Jahre früher publiziert hatte, wird hingegen von der Wissenschaftsgeschichte bezüglich des Klimawandeldiskurses kaum beachtet, zumindest wird ihm nicht der Stellenwert eingeräumt, der Tyndall heute zugeschrieben wird. Pouillets „Mémoire" beschäftigt sich mit dem Verhältnis von Absorption des Sonnenlichtes durch die Erdoberfläche und die Atmosphäre, mit dem Verhältnis von innerer („enceinte“) und äußerer („enveloppe“) Temperatur, mit den Temperaturen des Erdinnern und der Atmosphäre. Bemerkenswert erscheint, dass Agassiz das Wort Atmosphäre nicht begrifflich spezifiziert. Tyndall ging 1861 in seiner Veröffentlichung weiter und machte für die Erwärmung der Erde durch die Atmosphäre den Wasserdampf verantwortlich, der die Wärme des Sonnenlichts hindere, in den Weltraum auszutreten. „These waves dash in their passage against the atoms of oxygen and nitrogen, and against the molecules of aqueous vapour. ${ }^{\text {"51 }}$ Aber er schlüsselte die Bestandteile der Atmosphäre nach ihren Spurengasen auf: „It is well known that our atmosphere is mainly composed of the two elements oxygen and nitrogen. Theses elementary atoms may be figured as small spheres scattered thickly in the space which immediately surrounds the earth. They constitute about 99 1/2 per cent of the atmosphere. Mixed with theses atoms we have others of a totally

50 "The researches on glaciers which I have had the honour of submitting from time to time to the notice of the Royal Society, directed my attention in a special manner to the observations and speculations of De Saussure, Fourier, M. Pouillet, and Mr. Hopkins, on the transmission of solar and terrestrial heat through the earth's atmosphere." John Tyndall, On the Absorption and Radiation of Heat by Gases and Vapours, and on the Physical Connexion of Radiation, Absorption, and Conduction - The Bakerian Lecture, in: The London, Edinburgh, and Dublin Philosophical Magazine and Journal of Science 4, 1861, S. 169-194, hier: S. 169.

51 Tyndall, On Radiation (wie Anm. 21), S. 202. 
different character; we have the molecules, or atomic groups, of carbonic acid, of ammonia, and of aqueous vapour. ${ }^{\text {"52 }}$

Die Eiszeittheorie und der Klimawandel sowie die Bedeutung der Atmosphäre waren also bereits längst vor den Publikationen Arrhenius Diskursbestandteile. Sie gehörten seit $1840 \mathrm{zu}$ einem breit diskutierten und mit großer öffentlicher Anteilnahme begleiteten Phänomen des 19. Jahrhunderts. Johan Tyndall nimmt in der Tat eine besondere Rolle in diesem breiten Diskurs ein. Der Ire Tyndall war Bergsteiger und Gletscherforscher. Er bereitete die Erstbesteigung des Matterhorns vor. In den Waliser Alpen war er sogar namensgebend für den Pic Tyndall. John Tyndall versuchte das Matterhorn von Westen zu erklimmen. Er gelangte bis zu dem westlichen Seitengipfel und Grat des Matterhorns, der später nach ihm benannt wurde. Dort musste er umkehren. Erst Edward Whymper gelang die Erstbesteigung über eine andere Route. Doch die Kenntnisse der Schweizer Alpen genügten nicht, um seine Theorie zu komplettieren. Erst Tyndalls Beobachtungen in Irland ermöglichten eine weiterführende Argumentation. Bei Killarney fand er ähnliche Gletscherspuren wie in den Alpen an gegenwärtig bestehenden Gletschern. „Killarney, to which I have already referred, affords magnificient examples of ancient glacier action. No man with the slightest knowledge of the glacier operations of today could resist the conclusion, that the Black Valley of Killarney was once filled by a glacier fed by the snows form Magillicuddy's Reeks. " ${ }^{33}$ Tyndall war es durch seine glaziologischen Untersuchungen und seinen Kenntnissen in der Thermodynamik möglich, die Diskurse zusammenzuführen. Trotzdem sollten Agassiz' Texte nicht minder bewertet werden. Agassiz' und Tyndalls Texte weisen Positionen beider Diskurse auf, die des Klimawandels und des Treibhauseffekts. Beide liegen diskursiv sehr nahe beisammen, auch wenn Agassiz' Studie in der zweiten Hälfte des 19. Jahrhunderts an Wirkungsmacht in der wissenschaftlichen und öffentlichen Diskussion verloren hat und Tyndall sich als wegweisender Forscher durchsetzen konnte.

Auch ist eines gewiss: Der Klimawandel-Diskurs im ausgehenden 19. Jahrhundert ist isoliert von Glaziologie und Eiszeitforschung nicht aufzufinden. Eine stete Kohärenz der Themen ist auffällig und auch gar nicht abwegig. Es ist bemerkenswert, wie lange sich dieses Phänomen allein in den Diskursausschnitten erstreckt, die hier herangezogen wurden. Von den 1830er Jahren bis zum Ende des 19. Jahrhunderts. Dabei blieben noch einige wichtige Studien und Namen ihrer Autoren unerwähnt, die in das diskursive Umfeld gehören. So sei

52 Ebd., S. 201.

53 John Tyndall, Mountaineering in 1861. A vacation Tour, London 1862, S. $71 \mathrm{f}$. 
auf den Krakauer Professor für Erdkunde, Franz von Czerny, hingewiesen, der über die Veränderlichkeit des Klimas und ihre Ursachen 1881 publizierte. Seine Monographie ist ein umfassender Abriss des internationalen Forschungsstands zu Beginn der 1880er Jahre über die Gründe des Klimawandels. Für eine weiterführende Diskursforschung zur Frage des Klimawandels im 19. Jahrhundert ist sein Werk eine wichtige Referenzgröße. ${ }^{54}$ Auch die 1890 erschienen Studie des Berner Professors für Geologie Eduard Brückner ist hier zu nennen. Seine Studie über Klimaschwankungen seit 1700 gehört bis heute zu den umfassendsten und empirisch interessantesten Untersuchungen historischen Klimawandels. ${ }^{55}$ Brückners Ansatz bestand darin, die variierenden Wasserstände der Meere und Seen im Hinblick auf Klimaschwankungen und damit im Hinblick auf Klimawandel zu untersuchen. Beeinflusst von Brückner beschäftigte sich der bereits erwähnte italienische Meteorologe Luigi de Marchi in seiner Publikation von 1895 mit den Gründen der Eiszeit. ${ }^{56}$ De Marchi entwickelte im Anschluss an Pouillet mathematische Formeln, um den Klimawandel bezüglich der Eiszeiten berechnen zu können. Diese Arbeit ist für Arrhenius' Texte deswegen so wichtig, weil de Marchi keine Theorie entwickelte, die sich auf das Kohlenstoffdioxid in der Atmosphäre bezog. Die dafür notwendige Diskursposition lieferte erst Arrhenius, indem er die Erkenntnisse von Ernst Lecher mit dem Treibhauseffekt kombiniert. Mit Högbom und Arrhenius ist am Ende des 19. Jahrhunderts die Relation von Klima und Konsum nach einer langen diskursiven Genese ausgestaltet worden. Wie eingangs zu diesem Kapitel erläutert, verdeutlicht die Retrospektive in die Jahrhunderte davor, dass allein die Faszination der Gletscher als europäisches empirisches Anschauungsmaterial diese Korrelation von Klima und Konsum ermöglichten.

\section{Klimaphänomenologische Differenzerfahrungen in den Alpen}

Damit überhaupt Klimawandel diskursiv konstituiert werden konnte, bedurfte es bestimmter Auffälligkeiten, die sich aus Differenzerfahrungen ableiten ließen. Auf singuläre Wetterereignisse allein konnten sie sich nicht beziehen, sondern

54 Vgl. Franz von Czerny, Die Veränderlichkeit des Klimas und ihre Ursachen, Wien/ Pest/Leipzig 1881.

55 Vgl. Eduard Brückner, Klimaschwankungen seit 1700 nebst Bemerkungen über die Klimaschwankungen der Diluvialzeit, Wien/Olmütz 1890.

56 De Marchi, Le cause (wie Anm. 35), S. 209: „La temperatura dell' atmosfera quale è definita dalle nostre formole non si può ammettere come equivalente alla effettiva temperatura media dell' atmosfera, ossia alla media delle temperature corrispondenti alle diverse altezze, ma è una funzione assai più complessa." 
benötigten signifikante Häufungen von ähnlichen, in der Differenz zum Vorher außergewöhnlichen Wetterphänomenen. Das bedeutet, die Konstitution von Klimawandel besteht darin, dass er einen über Jahrzehnte währenden Reflex benötigte, um überhaupt eine Distinktion von Wetter und Klima zu ermöglichen. ${ }^{57}$ Nur regelmäßige und vor allem systematische sowie langwährende Wetteraufzeichnungen sowie die Speicherung von Wetterdaten hätten es zugelassen, generationenübergreifenden bestimmten Wetterphänomenen das Wort Klima, Klimawandel und deren Begrifflichkeiten zuzuschreiben. Diese systematischen Wetteraufzeichnungen gab es aber erst ab der zweiten Hälfte des 18. Jahrhunderts beispielsweise im Augustinerstift Rottenbuch, in Innsbruck, durchgeführt von Franz von Zallinger zum Thurn, und im Benediktinerkloster Kremsmünster. Reinhard Ferdinand Nießner geht in seinem Beitrag zu diesem Sammelband ausführlich darauf ein. ${ }^{58}$ Doch auch diese Wetterdaten reichten nicht dafür aus, dass Klimawandel in der Frühen Neuzeit hätte aus ihnen konstituiert werden können.

Trotz der weitgehend fehlenden systematischen Wettererfassung vor der zweiten Hälfte des 18. Jahrhunderts ist es möglich, durch phänomenologische Differenzerfahrung auf Klimawandel zu schließen. Allerdings ging dies nur an jenen Orten, an denen Klimawandel oder -schwankungen tatsächlich auch ästhetisch signifikante Differenzerfahrung zuließen. Besonders geeignet waren die anwachsenden oder abschmelzenden Gletscherzungen und Gletscherspuren, wie Findlinge, gekritzte Felsen sowie Moränen in den Alpen oder in deren Vorland. Zeitgemäße Beschreibungen dieser ästhetisch wahrnehmbaren Veränderungen der Gletscher lassen heute Klimaschwankungen in den vergangenen Jahrhunderten vor den Wetteraufzeichnungen am Ende des 18. Jahrhunderts erahnen. Der Grund für die Aufzeichnung von Wahrnehmungen der Gletscherausdehnung vor dem ausgehenden 18. Jahrhundert waren zufällige Erwähnungen in Urkunden. Meist regelten sie Besitz. Spätere Erwähnungen wurden durch Zerstörungen von Bauwerken oder das Überdecken von Weideland veranlasst. Gletscherzungen tangierten Eigentumsverhältnisse und hatten deswegen Einfluss auf Produktion und

57 Klima umfasst in seinem Begriff quantitativ viele ähnliche Wetter über einen langen Zeitraum, die eine Klimacharakteristik ergeben. Das Beispiel, das hier in diesem Beitrag verhandelt wird, ist die Ausdehnung der Gletscher in den Tiroler und Schweizer Alpen. Zu Klima und Wetter allgemein: Dieter Walch / Harald Frater (Hg.), Wetter und Klima. Das Spiel der Elemente - atmosphärische Prozesse verstehen und deuten, Darmstadt 2004.

58 Vgl. den Aufsatz von Reinhard Ferdinand Nießner in diesem Band über Franz von Zallinger zum Thurn und dessen Innsbrucker Wetteraufzeichnungen, die er bis zu seinem Tod durchgeführt hat. 
Konsum. Der größte österreichische Gletscher und zugleich der größte Gletscher der Ostalpen, die Pasterze unterhalb des Großglockners, führt den Klimawandel in ihrem Namen: Pasterze wird aus dem Slowenischen abgeleitet und verweist auf den Hirten (pastir). Eine entsprechende sagenhafte Erzählung, die das Motiv der Berner Blüemlisalp in sich trägt, schreibt der Pasterze zu, ehemals fruchtbarstes Weideland gewesen zu sein. ${ }^{59}$ Für das Anwachsen der Gletscher in der zweiten Hälfte des 16. Jahrhunderts und am Ende des 18. Jahrhunderts gibt es eine ganze Reihe von verschriftlichten Beobachtungen vor allem in Savoyen, der Schweiz und in Tirol.

\section{Gletscherphänomenologie und Glaziologie ab dem 12. Jahrhundert}

Heinz J. Zumbühl veröffentliche 1980 eine inzwischen prominente Studie über die Gletscherphänomenologie seit dem 12. Jahrhundert am Beispiel der beiden Grindelwaldgletscher. Er führte darin alle ihm verfügbaren Zeugnisse, Text wie Bild, über den Unteren und den Oberen Grindelwaldgletscher an. Die Sammlung Zumbühls lässt einige Diskurspositionen analysieren. Die mittelalterlichen Erwähnungen des Grindelwaldgletschers betreffen vor allem die Beschreibungen von Grundstücksangelegenheiten, welche die Lage von Grundstücken zu Nachbargrundstücken oder geologischen Auffälligkeiten in Relation setzen. So werden auch die Gletscherzungen der beiden Grindelwaldgletscher in einer Urkunde zwischen dem Augustiner Chorherrenstift Interlaken und den beiden Herren Walther und Conrad von Uspunnen (genannt Wädiswyl) erfasst, weil sie die Lage einer Alpe definiert. ${ }^{60}$ Damit sind die Gletscher ein Mittel zum Zweck der Lokalisierung von Besitz und Eigentum, aber die Beschreibung der Gletscher

59 Heinz J. Zumbühl, Die Klimaschwankungen der Grindelwaldgletscher in den historischen Bild- und Schriftquellen des 12. bis 19. Jahrhundert. Ein Beitrag zur Gletschergeschichte und Erforschung des Alpenraums, Basel 1980.

60 Als Beispiel diene die Lagebeschreibung der Alp Mettenberg: „Notum sit ergo universis presens scriptum inspecturis vel audituris, quod cum ego Wal. et $C$. frater meus, domini de Husponon, dicti de Wediswile, ex privilegio F. quondam imperatoris, patris H. imperatoris, ecclesie Interlacensi collato plene intellexerimus, terram cum monte qui dicitur Metenberch, que sita sunt in Grindelwalt a glacie inferiori usque ad superiorem, de jure pertinere ad ecclesiam Interlacensem, recognovimus nos mala fide possedisse. Unde jam dictam terram cum alpe ejusdem montis et omnibus appendiciis suis a glacie inferiori usque ad superiorem libere et absque omni conditione restituimus ecclesie jam dicte, nichil nobis juris in eisdem reservantes." Fontes Rerum Bernensium, Bd. 2, Bern 1877, S. 279. 
dient nicht zum unmittelbaren Zweck der Gletscherphänomenologie. Wichtiger erscheinen neben diesen Urkunden und Urbaren andere Zeugnisse, in denen der Untere Grindelwaldgletscher zum Aktanten wird. Wegen des ariden und heißen Sommers des Jahres 1540 zog sich der Gletscher merklich zurück. Drei Jahrzehnte später ab den 1570er Jahren veränderte sich die Situation und das Phänomen der Kleinen Eiszeit setzte sich klimatisch durch. Wolfgang Behringer verwies auf die Aufzeichnungen des Sebastian Schertlin von Burtenbach in diesen Jahren. ${ }^{61}$ Auch naturwissenschaftlich nachweisbar wuchsen seit den 1570er Jahren die Alpengletscher wieder an, nachdem selbst die Talböden der Grindelwaldgletscher als Weideland in der Mitte des 16. Jahrhunderts genutzt werden konnten. ${ }^{62}$ Der Verlust dieser Weideflächen schlug sich wiederum in Chroniken und Urkunden nieder, weil die Abweichung von Gewohnheiten Differenzerfahrungen ermöglichten. Als ein Abweichendes konnten sie schriftlichen Niederschlag finden. Zumbühl greift mitunter auf Franz Joseph Hugis, aber auch auf andere Berichte aus dem 18. und 19. Jahrhundert zurück, die wiederum auf Pfarrchroniken von Grindelwald ihre Erkenntnisse sammelten. ${ }^{63}$ Bemerkenswert ist das wohl erste literarische Denkmal, das einem Gletscher gesetzt wurde. Es sollte eine erstaunliche Rezeption erfahren. Der Pfarrer von Muri, Hans Rudolph Rebmann, dichtete in einem „poetischen Gespräch“ zweier Berge über den Unteren Grindelwaldgletscher und dessen Ausdehnung. ${ }^{64}$

61 Wolfgang Behringer / Hartmut Lehmann / Christian Pfister, Kulturelle Konsequenzen der „Kleinen Eiszeit“. Eine Annäherung an die Thematik, in: Dies. (Hg.), Kulturelle Konsequenzen der „Kleinen Eiszeit“. Cultural Consequences of the „Little Ice Age", Göttingen 2005, S. 7-27, hier: S. 7. Zum Egodokument: Sebastian Schertlin von Burtenbach, Leben und Taten des weiland wohledlen Ritters Sebastian Schertlin von Burtenbach, München 1910.

62 Über die dendrochronologische Analyse und Holzringanalyse von Baumstümpfen in Gletschermoränen datierte Ausdehnungen der Schweizer Gletscher: Hanspeter Holzhauser, Auf dem Holzweg zur Gletschergeschichte, in: Hallers Landschaften und Gletscher. Beiträge zu den Veranstaltungen der Akademien Schweiz 2008 zum Jubiläumsjahr „Haller 300“, in: Sonderdruck aus den Mitteilungen der Naturforschenden Gesellschaft in Bern 66, 2009, S. 173-208, hier: S. 185. Ab der Mitte der zweiten Hälfte des 16. Jahrhunderts dehnen sich die Schweizer Gletscher wieder aus, nachdem eine lange Abschmelzphase seit dem Beginn des Jahrhunderts zu beobachten war.

63 Hugi, Über das Wesen; Ders., Die Gletscher (beide wie Anm. 43).

64 Hans Rudolph Rebmann, Ein neuw, lustig, ersnthafft, poetisch Gastmal und Gespräch zweyer Bergen, in der löblichen Eydgenossenschafft und im Berner Gebiet gelegen: Nemlich deß Niesens und Stockhorns, als zweyer alter Nachbarn, Bern 1606. Zur Familie der Rebmann und der Entstehung des Werks: Hans Rudolf Scheidegger, „Ein 


\author{
„Bey Petronell am berg füwar \\ Ein grosser Glettscher hanget dar, \\ Hat gantz bedeckt dasselbig ort \\ Mit Heusren muss man rucken fort. \\ Stosst vor im weg das Erderich \\ Bäum Heuser Felsen wunderlich \\ Der Mettenberg an oberer Seit \\ Darauff das Schrickshorn sicht man weit \\ Hat auch sein Weiden b' sonderbar. \\ Der ander Glettscher obenhar \\ Der noch der grösser ist im Land \\ Damit würckt wunder Gotteshand."
}

Rebmanns „Petronell am berg füwar“ bezeichnet eine verwüstete Kapelle, St. Petronella, die bis zum ungewissen Zeitpunkt ihrer Zerstörung durch den Gletscher eine Wallfahrtskirche gewesen war. Die Verwüstung von St. Petronella und von einigen Bauernhäusern Grindelwalds war ein überregional bekanntes Phänomen des Gletscherwachstums, das bis in die Mitte des 17. Jahrhunderts immer wieder erzählt wurde und auch noch im 18. Jahrhundert von Johan Jakob Scheuchzer beschrieben wurde. In Matthäus Merians Topographia Helveticae von 1642 wird die Zerstörung und der Zustand des Grindelwaldgletschers ohne genaue Zeitangabe beschrieben. „Es mag dieser Berg, geliebter Leser, vor andern für etwas sonders und wohl für ein miraculum naturae gehalten werden. [...] Es ist nicht weit davon der Orth Capellen S. Petronel gewesen, [...] welchen Orth dieses Bergs Eygenschafft zum Wachsthumb sythero bedecket hat: Gestalt dann die Landleuthe dort herum observiren unnd bezeugen, daß dieser Berg dergestalt wachse unnd seinen Grund oder Erden vor sich her schiebe, daß wo zuvor eine schöne Matten oder Wiesen gewesen, dieselbe davon vergehe, unnd zum rauhen wüsten Berg werde. Ja an etlichen Orten man ime ums dieses seines Wachsthumbs willen, mit denen darauf und daran gestanden Bawren Häuser oder Hütten, haben weichen müssen: Es wachsen auch auß ihm grosse ruhe Schrollen oder Eyßschlupen, wie auch Steine, unnd ganze Felsenstück, die der Orths befindliche Häuser, Bäume und anders von sich beyseits in die Höhe schieben." ${ }^{65}$ Die Ausdehnung des Unteren Grindelwaldgletschers setzte sich, so lässt sich Merian interpretieren, bis zum Erscheinen der „Topographia

poetisch Gespräch zwischen Niesen und Stockhorn..., in: Jahrbuch vom Thuner und Brienzersee 1994, S. 143-152.

65 Matthäus Merian, Topographia Helvetiae, Rhaetiae, Et Valesiae, Das ist, Beschreibung und Eigentliche Abbildung der Vornehmbsten Stätte und Plätz in der Hochlöblichen Eydgenoßschafft, Graubünden, Wallis, und etlicher Zugewandten Orten, Frankfurt a.M. 1642, S. $26 \mathrm{f}$. 
Helvetiae" 1642 fort. Aber Merian ist bekannt dafür, dass seine Beschreibungen auf frühere lokale Texte und Ansichten zurückgehen. Deswegen sollte nicht allzu viel auf das Jahr 1642 gegeben werden. Jedoch hat Hanspeter Holzhauser mit Dendrochronologie und der C14-Methode bestätigt, dass die Gletscherausdehnung von den 1570er Jahren fast ein Jahrhundert bis 1678 andauerte. ${ }^{66}$ Auffällig weist Merians Text einige Ähnlichkeiten mit dem Gedicht von Hans Rudolph Rebmann und auch mit dem seines Sohns Valentin Rebmann neu herausgegebenen Gedicht von 1620 auf. Nahe liegen die Textbestandteile und Positionen zusammen.

„Im Erdtreich wachst er immerdar,

Das Landleut klarlich nemmen war,

Wie er den grund stosst fürer bass,

Da vor ein schöne Matte was,

Ist jetzt grewlich wüst bedeckt,

Mit Gletscher, der offt kracht und kleckt,

Wirfft thüffe Schründ, herd, sand, und stein,

Holtz, und was sonst für wust gemein,

Würffts auss Morgens und Abends zwar,

Wan es in heyssen tagen war,

Gwint er sein spält und schründ mit krache,

Als wolt er einen Donner machen,

Also wachsst er fürbas all Jahr. ${ }^{" 67}$

Der Berg/Gletscher als Gottes/Naturwunder.

Merian: „Es mag dieser Berg vor andern für etwas sonders und wohl für ein miraculum naturae gehalten werden."

Hans Rudolph Rebmann: „Der noch der grösser ist im Land / Damit würckt wunder Gotteshand.“

St. Petronell zerstört.

Merian: „Capellen S. Petronel gewesen, [...] welchen Orth dieses Bergs Eygenschafft zum Wachsthumb sythero bedecket hat."

Hans Rudolpf Rebmann: „Bey Petronell am berg füwar / Ein grosser Glettscher hanget dar / Hat gantz bedeckt dasselbig ort."

66 Holzhauser, Holzweg (wie Anm. 62), S. 185. Holzhausers Befund steht in gewisser Weise im Widerspruch zum Maunderminimum, das von 1645-1715 angenommen wird. Der Grund für die Gletscherausdehnung im Alpenraum kann folglich nicht nur auf die Sonnenfleckenaktivität zurückgeführt werden.

67 Zitiert nach: Zumbühl, Klimaschwankungen (wie Anm. 59), S. 18. 
Landleute nehmen Wachstum an überdeckten Wiesen wahr.

Merian: „(1) Gestalt dann die Landleuthe dort herum observiren unnd bezeugen, (2) daß dieser Berg dergestalt wachse unnd (3) seinen Grund oder Erden vor sich her schiebe, (4) daß wo zuvor eine schöne Matten oder Wiesen gewesen, dieselbe davon vergehe, unnd zum (5) rauhen wüsten Berg werde."

Hans Rudolph Rebmann: „(3) Stosst vor im weg das Erderich.“

Valentin Rebmann: „(2) Im Erdtreich wachst er immerdar / (1) Das Landleut klarlich nemmen war / Wie er den grund stosst fürer bass / (4) Da vor ein schöne Matte was / (5) Ist jetzt grewlich wüst bedeckt."

Häuser müssen neugebaut werden.

Merian: „Ja an etlichen Orten man ime ums dieses seines Wachsthumbs willen, mit denen darauf und daran gestanden Bawren Häuser oder Hütten, haben weichen müssen."

Hans Rudolph Rebmann: „Mit Heusren muss man rucken fort/ Bäum Heuser Felsen wunderlich"

Vielmals beruhen die Beschreibungen und Stiche von Merian auf einer guten Bibliotheksrecherche. Deswegen wäre die Beschreibung von 1642 allein kein Indiz für das Anwachsen des Unteren Grindelwaldgletschers, weil sie sich sehr offensichtlich auf die Gedichte von 1606 und 1620 bezieht. Dies belegen die Ähnlichkeiten der Diskurspositionen Rebmanns und Merians. Damit ist eine berühmte Stelle von Johann Jakob Scheuchzer ebenso von ihrer Datierung problematisch, denn Scheuchzer verweist darin explizit auf Merian: „Addunt ex Mariani Topograph. Helv. p. 26. motum veluti progressivum quo terminos suos magis magisque soleant protendere, et esempli loco afferunt Divae Petronellae sacellum, in Grindelia valle, glacie totem opertum, et sede sua depulsum, quae adhuc dum digitis demonstrari solet, terram item adjacentem, una cum arboribus, casis stabilisiert et pascuis remoras, ut insola aliorsum casas suas migrare dependentium causa non miraculo alicui, quid verum physicarum imperiti somniant, sed omnio causi naturalibus adsorbii debet. ${ }^{\text {"68 }}$ Scheuchzer bezieht sich also keineswegs auf das Jahr 1642, sondern entweder auf 1606 oder, sollte der Zustand des Unteren Grindelwaldgletschers auch 1620 angehalten haben, auf letzteres Datum. Jedoch sollte diese Datierungsfrage nicht den Beitrag schmälern, den Johann Jakob Scheuchzer mit seinem umfangreichen Werk „Ouresiphoites Helveticus, sive itinera per Helvetiae alpinas

68 Johann Jakob Scheuchzer, Ouresiphoites Helveticus, sive itinera per Helvetiae alpinas regions, 4 Bde., Leyden 1723, hier: Bd. 4, S. 287. 
regiones" im Jahr 1723 herausbrachte. Die Ausgabe von 1723 vereint Reisen und Exkursionen des Naturwissenschaftlers aus den Jahren 1701-1711 in vier Bänden und verzeichnet die Schweiz als erste große und sehr genaue Landesbeschreibung, die kultur- aber vor allem naturwissenschaftliche Aspekte berücksichtigt. Für die Gletscherforschung ist vor allem der zweite Band von Interesse, der thematisch Gletscherbeschreibungen und das von Scheuchzer gesammelte Wissen über die jeweiligen Schweizer Gletscher umfasst. Wie sein gesamtes Werk haben auch diese Landesbeschreibungen enzyklopädischen Charakter. Systematisch sammelt er sie und verweist auf die Literatur seiner Zeit: So definiert er beschreibend, was Gletscher seien und wie sie entstünden, sogar auf eine mögliche etymologische Herkunft des Namens „Alpen“ weist er - Alpes, Alpos, Albos -, weil die Berge ewig weiß erschienen. ${ }^{69}$ Die Ästhetik dieser weißen Berge mache die Alpen so anziehend. „Causas admirandi hujus Phaenomeni quod nos media etiam aestate summa cum delectatione intuemur quotidie, explicuimus alibi. "70 Auf den Seiten 281 bis 286 referierte Scheuchzer die Forschungsgeschichte bezüglich der Gletscher. Dort bezieht er sich vor allem auf Johannes Heinrich Hottinger, der eine Beschreibung der Schweizer Gletscher vor Scheuchzer im Jahre 1706 vorgelegt hatte. ${ }^{71}$ Ebenso verweist Scheuchzer auf Rebmanns Gedicht über die Grindelwaldgletscher und auf die schon erwähnte Beschreibung Matthäus Merians. Für Scheuchzer sind Gletscher wie auch für Rebmann und Merian ein Wunder oder gar ein Gotteswunder, das sich verändert, das einer Naturgewalt wie einem Fluss gleicht, dem sich die Baugrenze nicht zu sehr nähern sollte. In diesem Diskurs liegt auch Albrecht von Hallers Gedicht „Die Alpen“. Sein Panegyrikus und Scheuchzers Beschreibung und systematische Erfassung der Schweiz sind beide auf ihre Weise verantwortlich, dass der Alpendiskurs und das ewige Eis auf den Bergen zu mächtigen Diskursbestandteilen des 18. und 19. Jahrhunderts wurden. ${ }^{72}$ Aber ein weiterführender Aspekt, beispielsweise ein exzeptioneller oder spektakulärer Klimawandel, findet sich bei diesen Autoren noch nicht. Dieser Schritt vollzieht sich erst in den 1740er Jahren.

1742 bereiste der Genfer Pierre Martel das Tal von Chamonix. Von dieser Reise blieb der Text: „Voyage aux glacières du Faucigny“. Martel verfasste ihn als Brief

69 „Montes perpetua Nive tectos, Alpes, quasi Alpos seu Albos, ex quorundam Philologorum mente dici constat." Ebd., Bd. 2, S. 280.

70 Ebd., S. 281.

71 Johannes Heinrich Hottinger, Descriptio montium glacialium Helveticorum, Zürich 1706.

72 Albrecht von Haller, Alpen, in: Ders., Hallers Versuch Schweizerischer Gedichten, Bern 1732, S. 1-25, hier: S. 17. 
an William Windham, der 1741 dieselbe Reise unternommen hatte. Der Genfer beschrieb, wie er die Gletscher und deren Umgebung untersuchte und feststellte, Gletscher wachsen in bestimmten Epochen an, in anderen schmelzen sie ab. „Les glacières et la vallée de glace augmentent et ne sont pas toujours dans le même état. Les glaces augmentent ou diminuent suivant le temps. Il y a apparence qu'elles ont été beaucoup plus abondantes. Il y a lieu de croire qu'elles ont du avoir plus de 80 pieds au dessus du lieu où elles sont actuellement, par les vestiges qui y sont restés. "“73 („Die Gletscher und das Eistal nehmen zu und sind nicht immer in demselben Zustand. Das Eis nimmt mit der Zeit zu oder ab. Es scheint, dass sie sich stark zurückgezogen hätten. Es gibt durch die Spuren Grund zu der Annahme, dass sie mehr als 80 Fuß über dem Ort gewesen sein müssen, wo sie aktuell sind.“)

Dreißig Jahre nach Martel bereiste Gottlieb Sigmund Gruner die helvetischen Gletscher. Auch seine Reise brachte einen Text hervor: „Reisen durch die merkwürdigsten Gegenden Helvetiens", London/Bern 1778. Sein Werk ist wesentlich umfangreicher als das von Martel. Es stellt eine Bestandsaufnahme der Schweizer Gletscher in den 1770er Jahren dar. Auch in Gruners Text wird historischer Wandel der Gletschergrößen konstatiert. So sei der zur Reise Gruners gegenwärtige Zustand des Rhonegletschers 1778 kleiner als jene Ausdehnung, die in den Kupferstichen von Johann Jakob Scheuchzer festzustellen sei, deren Vorlagen der Naturforscher während seiner Reise etwa 1710 als Zeichnungen anfertigen ließ und in seinem „Ouresiphoites Helveticus, sive itinera per Helvetiae alpinas regiones" als Kupferstiche hat veröffentlichen lassen. ${ }^{74}$

Einen Kupferdruck, den Gruner in seinem Text erwähnte, stellt den Rhonegletscher dar. Der Gletscher scheint aus zwei Teilen zu bestehen. Der obere Teil zeigt eine zerklüftete Gletscherzunge, die aus den Bergen herabfallend brüchig, mit kleinen herauskragenden sowie chaotisch anmutenden Eispyramiden wie ein kleines Eisgebirge wirkt. Gipfel um Gipfel drängt sich dicht an dicht. Der Zeichner, auf dessen Vorlage der Kupferdruck zurückgeht, stand frontal zum Gletscher. Rechts im Bild wird der Gletscher durch einen Berg eingeschränkt, der in der Legende als Mont Furca bezeichnet wird. Die kleinen Eisgipfel des Gletschers enden in einer haubenartigen Viertelkugel, wohl eine Art Schutthügel aus Eis, in den die Gletscherzunge mit ihren zerklüfteten Eisgipfeln am Talgrund übergeht. Jedoch wird nicht erkenntlich, wie diese Bestandteile des Gletschers zusammengehören. Ein Bachlauf tritt aus dieser Viertelkugel aus. Er vereinigt sich

73 Pierre Martel, Voyage aux glacières de Faucigny 1742, in: William Windham / Ders., Relations de leurs deux voyages aux glaciers de Chamonix, Genf 1879, S. 35-68, hier: S. 48 .

74 Scheuchzer, Ouresiphoites Helveticus (wie Anm. 68), Bd. 2, S. 278. 
mit einem weiteren Bachlauf zur Rhone. Dieser Befund des Kupferstichs wird von einem Gemälde von William Pars von 1770/71 bestätigt. Pars wählte nicht den frontalen Blick von Süden nach Norden, sondern von West nach Ost zum Furka hin, der den Hintergrund seines Gemäldes bildet. Seinen Standpunkt wählte er direkt vor der haubenartigen Viertelkugel, die allerdings weniger mächtig wirkt als bei Scheuchzer. In dieser Ansicht wird deutlich, um was es sich dabei handelt. Diese Art des Gletschers, der eine Kugel oder einen Fächer ausbildet, heißt Vorlandgletscher. Die zerklüftete Gletscherzunge, die zusammen mit der Viertelkugel den Vorlandgletscher bildet, vollzieht einen diagonalen Bildaufbau. Pars Gemälde entstand annähernd zu dem Zeitpunkt, als Gruner die Schweizer Alpen bereiste. Seine Darstellung des Rhonegletschers wirkt sehr real-mimetisch. Ein direkter sowie naiver Abgleich dieser Ansicht mit dem Kupferstich Scheuchzers scheint einen eindeutigen Befund zu ergeben: Der Rhonegletscher hat in der Zeit von 1710-1770 an Ausdehnung verloren. Aber eine gewissenhafte Aussage darüber lässt sich nicht treffen, weil der Kupferstich eine ähnliche Perspektive benötigte wie das Gemälde von Pars - oder umgekehrt.

Einen gegenteiligen Befund zum Abschmelzen des Rohnegletschers stellte Gruner am Finsteraargletscher fest. Während unbestimmbarer Zeit sei der Gletscher angewachsen. Auf mündliche Überlieferung berufend, schrieb er, es werde erzählt, ehemals sei der aktuelle Ort eisfrei gewesen und Blüemlisalp genannt worden. ${ }^{75}$ Auch den Grindelwaldgletscher beschreibt Gruner als ein sich wandelndes Gebilde, das durch Schwund und Wachstum gekennzeichnet sei. Wiederum bezieht er sich explizit auf Scheuchzers Abbildung. ${ }^{76}$ Der Gletscher sei deswegen so interessant, weil er so oft in Kupfer gestochen worden sei. Deswegen könne an den verschiedensten Abbildungen des Gletschers Schwund und Wachstum abgeschätzt werden. „Keinen, von allen Gletschern, in der Welt ist so viel Ehre bewiese worden, als diesem. Er ist nicht nur von vielen beschrieben, sondern auch zu vielen malen in Kupfer vorgestellt worden. Diese Kupfer, die den Gletscher auf sehr verschiedene Weise vorstellen, dienen aber dazu, den verschiedenen und abwechselnden Anwachs und Abnahm desselben zu zeigen. Die einzelne Zeichnung, [...] stellt denselben vor, wie er sich vor etwa 50. Jahren befunden hat. Damals war er größer als jemals, und hatte einen ungeheuren Eisstock an seinem Ende; obenher aber war er beynahe eben. [...] Das größere Kupfer oder die Aussicht im Grindelwald, die diese beyde Gletscher in der Ferne zeigt, und etwa 20. Jahr vorher mag

75 Gottlieb Siegmund Gruner, Reisen durch die merkwürdigsten Gegenden Helvetiens, 2 Bde., London/Bern 1778, 1. Teil, S. 249.

76 Scheuchzer, Ouresiphoites Helveticus (wie Anm. 68), Bd. 3, S. 482. 
gemacht worden seyn, stellt denselben vor, wie er zu selbiger Zeit gewesen; und ungefehr anjezo ist. " 77

Der bereits mehrfach erwähnte Horace-Bénédict de Saussure erhob ebenfalls eine gewichtige Stimme im Gletscherdiskurs. Vor allem die Frage der Schwankungen der Gletschergröße beschäftigte ihn. De Saussure nimmt im 18. Jahrhundert die Schlüsselstellung ein, in dem die beiden Diskurse, Klimawandel bezogen auf Gletscher und Grundlagen, mit denen der Treibhauseffekt durch Fourier entdeckt werden konnte, zusammenlaufen. Ähnlich wie John Tyndall im 19. Jahrhundert war er beides, Bergsteiger und Naturforscher, beziehungsweise Naturwissenschaftler. Der für den Gletscherdiskurs wohl wissenschaftlichste Text des 18. Jahrhunderts findet sich im ersten Band seiner vierbändigen Reiseberichte „Voyages dans les Alpes", veröffentlicht von 1779-1796. ${ }^{78}$ Seine Texte weisen mehrere Diskurspositionen auf, die teils schon von anderen Autoren bekannt sind.

1. Gletscher vergrößern und verkleinern sich periodisch.

„Léxistence des périodes est un fait certain [...].“79

2. Es gibt jedoch keine regelmäßigen Perioden, die außerhalb der Köpfe der Menschen existierten.

„leur [les périodes] régularité seule est imaginaire; mais comme on le fait, la régularité plait aux hommes, elle semble leur assujettir les événemens, \& ce nombre mystérieux de deux fois sept années, assez grand pour que le souvenir de l'état précis des chose se soit effacé de la mémoire de ces bonnes gens qui ne tiennent aucun registre, a pu facilement trouver créance dans leurs esprits. “" („Ihre Regelmäßigkeit ist imaginär. Aber wie es eben ist, die Regelmäßigkeit gefällt den Menschen. Es scheint, sie unterwerfen Ereignisse unter diese Regelmäßigkeiten, und diese geheimnisvolle Zahl von zweimal sieben Jahren, ist groß genug damit die Erinnerung an den genauen Zustand der Dinge aus dem Gedächtnis dieser Männer ausgelöscht wird. Gute Leute, die keine Aufzeichnungen führen, können in ihren Köpfen leicht Glaubwürdigkeit finden.")

3. In früheren Zeiten müssen die Gletscher wesentlich ausgedehnter gewesen sein.

„Le grand glacier des Bois, dans la vallée de Camouni, a eu indubitablement ses glaces anciennement plus hautes \& plus étendues quelles ne le sont aujourd'hui. Car au dessous de Montanvert, ces glaces sont de 40 ou 50 pieds plus basses que cet amas de débris qui borde le glacier, \& que lon nomme la moraine. Elles doivent pourtant avoir été de niveau

77 Gruner, Reisen (wie Anm. 75), 2. Teil, S. 13 f.

78 De Saussure (wie Anm. 42), Voyages.

79 Ebd., Bd. 1, S. 463.

80 Ebd. 
avec ces débris, \& accumulés dans cette place; ce ne sont point des fragments détachés de la montagne même de Montanvert, mais de Granits en masse dont on ne voit des montagnes qu'au haut de la vallée de glace. Et au bas du même glacier, au Nord-Ouest de la sortie de l'Arvéron, on voit jusques sur un grand rocher calcaire, dont je donnerai la description, des blocs de Granit, déposés anciennement par le glacier, qui est aujourd'hui fort en arrière de rocher. “81 („Der große Glacier des Bois im Camouni-Tal [Tal von Chamonix] hatte zweifellos sein Eis, das früher höher und umfangreicher war als heute. Denn unterhalb von Montanvert sind diese Eisschichten 40 oder 50 Fuß tiefer als dieser Trümmerhaufen, der den Gletscher begrenzt und Moräne genannt wird. Sie müssen jedoch auf gleicher Höhe mit diesen Trümmern gewesen sein und sich an dieser Stelle angesammelt haben; es sind keine Fragmente, die vom Berg Montanvert gelöst sind, sondern Granitmassen, die nur im oberen Teil des Eistals in den Bergen zu sehen sind. Und auf dem Boden desselben Gletschers, nordöstlich des Arveron Austritts [Arveyron Austritt ins Tal von Chamonix], kann man auf einem großen Kalkfelsen, den ich beschreiben werde, Granitblöcke sehen, die früher vom Gletscher, der heute weit hinter dem Felsen ist, abgelagert wurden.")

4. Methode: Es bedarf der Sammlung von exakten Daten über einen langen Zeitraum, um Aussagen über Wachstum, Stillstand oder Abnahme von Gletschern machen zu können.

„Ce ne sera qu’après avoir rassemblé beaucoup de faits, \& les avoir comparés avec une grande exactitude pendant une longue suite d'années, que l’on pourra décider avec certidude, si la masse totales glaces augment, diminue, ou demeure constamment la même. “82 („Erst wenn man viele Tatsachen gesammelt und sie über eine lange Reihe von Jahren mit großer Genauigkeit verglichen hat, wird es mit Sicherheit möglich sein, zu entscheiden, ob die gesamte Eismasse zunimmt, abnimmt oder sogar konstant bleibt.“)

\section{Grund für das Abschmelzen von Gletschern ist die innere Wärme der Erde.}

„L'une de ces causes est la chaleur intérieure de la Terre, qui fait fondre les neiges \& les glaces, même pendant les froids les plus rigoureux, lorsque leur épaisseur est assez grande pour

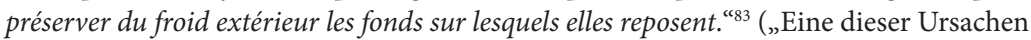
ist die innere Hitze der Erde, die Schnee und Eis schmilzt, selbst während der kältesten Stürme, wenn ihre Dicke groß genug ist, um die Untergründe, auf denen sie ruhen, vor der äußeren Kälte zu schützen.")

Horace-Bénédict de Saussure ist der aufgeklärte Forscher der Gletscherphänomenologie und wahrscheinlich auch, bezogen auf die hier analysierten Texte, der erste Glaziologe, der voreilige Erklärungen und Aussagen meidet.

81 Ebd., S. $464 \mathrm{f}$.

82 Ebd., S. 465.

83 Ebd., S. 451, § 532. 
Diskursposition 1 stimmt bezogen auf den Diskurs des 17. und 18. Jahrhunderts mit der Gletscherphänomenologie überein: Es ändert sich etwas bei den gegenwärtigen Gletschern an ihrer Ausdehnung. Ob in den Gedichten der beiden Rebmanns, Matthäus Merians Beschreibung in der Topographia, Scheuchzers wiederholende Verweise auf Merian oder durch den Gletscherphänomenologen Gruner, diese Position ist über zwei Jahrhunderte nachweisbar.

Diskursposition 2 ist eine Position der Aufklärer, die sich nicht selten über die Doxa der nicht aufgeklärten Menschen erheben. Die regelmäßigen periodischen Änderungen - sieben Jahre Ausdehnungen und sieben Jahre Zurückziehen der Gletscher - werden in das Reich der Sagen, Mythen und Legenden gerückt, die sich auf ihre Erinnerung, nicht auf Aufzeichnungen und klares Zahlenmaterial berufen. Martel hatte bereits die Diskursposition des periodischen Zunehmens und Abnehmens beschrieben. ${ }^{84}$ Auch Martel schreibt nur von „suivant le temps“ - jedoch nicht von einer geregelten starren Periodizität. Die Doxa der siebenjährigen Periodizität ist im Alpenraum weit verbreitet gewesen. Die zentrale Figur der Tiroler Gletscherphänomenologie und -forschung des 18. Jahrhunderts, Joseph Walcher, der die Tiroler Ferner (Gletscher) besuchte, erhob sich ähnlich über die Doxa der Bergbewohner. Mit ihm war ebenso ein ausgebildeter Naturforscher in den Alpen, vor allem im Bereich der Ötztaleralpen unterwegs, der dezidiert zur Gefahreneinschätzung von Seiten der Habsburger Administration geschickt worden war. Er vertrat folgende Positionen zur Periodizität: „Von dem Wachsthume der Ferner hört man in Tyrol fast so vieles Fabelhaftes, als in der Schweiz von dem Wachsthume der Gletscher, von welchen man vormals geglaubt hat, daß sie durch sieben Jahre allezeit $z u$, und durch andre sieben Jahre wiederum abnehmen. Allein beides, das Abnehmen sowohl, als der Wachsthum hängt nur von der Witterung $a b$, welche in verschiedenen Jahresläuften sehr ungleich ist. Die Ferner nehmen ab, wenn das Eis schmelzet: sie wachsen, wenn entweder durch eine große Kälte ein neues Eis hervorgebracht, oder das in den Thälern sich schon befindende Eis durch die aus den Bergen herabstürzenden Eisstücke mehr aufgehäufte, aber das gar zu viel aufgehäufte von dem noch nachfolgenden fortgeschoben, und sich weiter zu verbreiten gezwungen wird." ${ }^{85}$

Walcher geht also von einer Theorie der externen Wärme und Kälte aus, die für die Formation des Gletscherumfangs verantwortlich seien. Er beschäftigte sich in den Ötztaleralpen übrigens mit einem Phänomen, das zur momentan frühesten bekannten bildlichen Gletscherdarstellung führte, dem Rofener Eissee. Dieser

84 Martel, Voyage (wie Anm. 73), S. 48.

85 Joseph Walcher, Nachrichten von den Eisbergen in Tyrol, Frankfurt/Leipzig 1773, S. 5. 
Eissee entstand - periodisch, aber ohne Regelmäßigkeit. Eine Mauer aus Eis, die vom Vernagtferner gespeist wurde, staute die Rofener Ache, die aus dem Hintereisferner austrat. Am 20. Juli 1600 brach diese Staumauer, die während der Kaltphase um 1600 entstanden war. Die Flutwelle wirkte vernichtend auf das Ötztal. Sie und vor allem die diskursive Flutwelle, die sich nach diesem Ereignis über ganz Europa verteilte, führte zur Beschäftigung mit den Gletschern (beziehungsweise Ferner), die zuvor, wie von Zumbühl dargestellt, vor allem für die Grenzziehung und somit zur Lokalisierung von Grundstücken notwendig gewesen waren. Der Rofener Eissee, der mit jeder periodisch eintretenden übermäßigen Ausdehnung des Vernagtferners bis ins 19. Jahrhundert hinein immer wieder entstand, gilt als der Beginn der Gletscherphänomenologie und der Glaziologie. Die Katastrophe führte auch zur ersten bildlichen Darstellung eines Alpengletschers von Abraham Jäger, der in einer Tinten- und Aquarellzeichnung den im Jahr 1601 wieder befüllten Rofener Eissee festhielt. ${ }^{86}$

Das Anwachsen der Gletscher um 1773 wird von Joseph Walcher auch mit dem Blüemlisalp-Narrativ beschrieben, wie es sich bei Gruner findet, aber auch an der Pasterze: Viehweiden seien mit Eis überdeckt, der Weinbau nicht mehr in bestimmten Höhenlagen möglich. ${ }^{87}$

Diskursposition 3 unterscheidet sich bei Horace de Saussure von Martel nur durch den Ort der Beobachtung. Für Martel sind es die Gletscher von Faucigny im nördlichen Montblanc-Massiv. Für de Saussure finden sich die Beispiele in direkter Umgebung von Chamonix.

Martel: $\quad$ „Il y a lieu de croire qu'elles ont du avoir plus de 80 pieds au dessus du lieu où elles sont actuellement, par les vestiges qui y sont restés. " ${ }^{88}$

De Saussure: „Car au dessous de Montanvert, ces glaces sont de 40 ou 50 pieds plus basses que cet amas de débris qui borde le glacier, \& que l’on nomme la moraine. Elles doivent pourtant avoir été de niveau avec ces débris, \& accumulés dans cette place. " ${ }^{\text {"9 }}$

86 Zum Vernagt ferner im diskursiven Feld des 19. Jahrhunderts: Michael Stotter, Die Gletscher des Vernagtthales in Tirol und ihre Geschichte, Innsbruck 1846; Eduard Richter, Zur Geschichte des Vernagtgletschers. Beiträge zur Geschichte und Geographie der Alpen IV, in: Zeitschrift des Deutschen und Österreichischen Alpenvereins 7, 1877, S. 164-168.

87 Walcher, Nachrichten (wie Anm. 85), S. 6.

88 Martel, Voyage (wie Anm. 73), S. 48.

89 De Saussure, Voyages (wie Anm. 42), Bd. 1, S. 464 f. 
Diskursposition 4 ist genuin für de Saussure im Vergleich zu den hier besprochenen Texten. Ganz Naturforscher, setzt er auf eine geregelte, klar strukturierte empirische Grundlage. Zahlreiche Messungen über einen langen Zeitraum seien notwendig, um valide Aussagen über generelles Wachstum, Kontinuität oder über Abnahme der Gletscher machen zu können.

Diskursposition 5 betrifft einen der Gründe für das Abschmelzen der Gletscher. Dieser Grund sei die stete innere Wärme der Erde. De Saussure erklärte diese innere Wärme der Erde folgendermaßen: „Notre Terre a reçu du Soleil, \& peut-être d'autres causes qui ne nous sont pas bien connues, un certain degré de Chaleur, qui pass pour être uniforme à la profondeur de 60 ou 80 pieds dans les parties solides de ce Globe; \& qui dans ces mêmes parties à cette même profondeur, n'est pas sensiblement affecté par les variations des saisons. Cette chaleur est ce que jappelle la chaleur intérieure de la Terre. ${ }^{“ 90}$ („Unsere Erde hat von der Sonne und vielleicht auch von anderen Ursachen, die uns nicht bekannt sind, ein gewisses Maß an Wärme erhalten, das in den festen Teilen dieses Globus in einer Tiefe von 60 oder 80 Fuß gleichmäßig ist; und die in diesen gleichen Teilen in derselben Tiefe, durch die Schwankungen der Jahreszeiten nicht bemerkenswert betroffen ist. Diese Hitze nenne ich die innere Wärme der Erde.“) Diese Auffassung von der Erwärmung der Erde ohne Atmosphäre und Treibhauseffekt entspricht den Diskurspositionen der Thermodynamik seiner Zeit. Johann Heinrich Lambert, der sein weit verbreitetes thermodynamisches Werk „Pyrometrie“ 1779 veröffentlichte, formulierte ähnlich: „Die Wärme, welche die Erde von der Sonne erhält, zieht sich in dieselbe hinein, und schon aus diesem Grunde wird sie an der Oberfläche schwächer als sie sonst seyn würde. Es geht nun hingegen auch von der Wärme, so die Oberfläche hat, immer ein Theil durch die Luft in die Höhe, wo sie sich, so zu sagen, verliert. Dieser Abgang dauert Tag und Nacht in einem Fort, und ist dato stärker, je wärmer die Erdfläche ist, je kürzer die Tage und je länger die Nächte

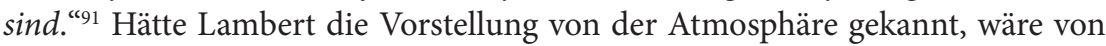
keinem Wärmeverlust die Rede. Die Idee des Hineinziehens der Sonnenwärme in den Erdkörper scheint die vorherrschende Diskursposition des ausgehenden 18. Jahrhunderts gewesen zu sein. Die Atmosphäre muss im Diskurs zwischen Lamberts „Pyrometrie“ von 1779 und Fouriers „Mémoire“ von 1827 konstituiert worden sein. ${ }^{92}$ Eine genauere Lokalisierung war mir bislang nicht möglich.

90 Ebd., S. 451.

91 Johann Heinrich Lambert, Pyrometrie, Berlin 1779, S. 322.

92 Bislang konnte ich die Diskursposition von der Existenz der Atmosphäre nicht fixieren. Fourier ist sich der Atmosphäre in seinem Mémoire sicher. Also muss diese Position 
Die Vorabbeurteilung de Saussures Stellung in dem Gletscherdiskurs bleibt bestehen. Er ist ein aufgeklärter Naturforscher, der sich auf das Wissen seiner Zeit stützt und gleichzeitig dieses Wissen auf eine empirisch gediegene Basis stellt. In seinen thermodynamischen Überlegungen steht er einem der bekanntesten Physiker seiner Zeit, Johann Heinrich Lampert, nicht hinterher. Obgleich de Saussure das Heliothermometer (das von Fourier beschriebene treibhausartige Gefäß) entwickelte, konnte er die Bauart nicht mit dem Aspekt der Atmosphäre verknüpfen, weil er sie nicht kannte. Die Grundlage für Fourier war sein Werk, aber die Kombination von Gletscherdiskurs und atmosphärischem Treibhauseffekt blieb ihm verwehrt. Obwohl er ähnliche Dispositionen wie John Tyndall, die des Bergsteigers und Naturforschers, vorweisen konnte, hätte er den anthropogenen Einfluss auf die Gletscherzunahme beziehungsweise -abnahme noch nicht herstellen können. De Saussure war entscheidend für die Entwicklungen im 19. Jahrhundert. Seine Texte nehmen zentrale Schlüsselpositionen ein, verbleiben aber - und das ist nicht erstaunlich - in den Diskursen ihrer Zeit.

\section{Zusammenfassung zum Diskurs ab 1700}

Johann Jakob Scheuchzer und auch Albrecht von Haller mit seinem fulminanten Gedicht „Die Alpen“ sind beide auf ihre Weise verantwortlich, dass der Alpendiskurs und das ewige Eis auf den Bergen zu mythischen Narrativen des 18. und 19. Jahrhunderts wurden. ${ }^{93}$ Phänomene eines noch opaken Wandels waren durch Gruner und Martel in den Gletscherdiskurs im 18. Jahrhundert eingespeist worden. Martel gewann seine Erkenntnis aus materiellen Spuren oberhalb der damals gegenwärtigen Gletscherzunge, Gruner hingegen aus dem Vergleich mit Abbildungen und mündlichen Erzählung, denen er offensichtlich großes Vertrauen entgegenbrachte. Hallers Gleichsetzung der Berge mit Edelsteinen mag dafür ein Beispiel sein: „Den ein verjährtes Eiß dem Himmel gleich getürmt / Sein frostiger Krystall schickt alle Strafen wieder / Den die gestiegene Hits im Krebs umsonst bestürmt. ${ }^{\text {" } 94}$

Für den Tiroler Joseph Walcher gilt, dass er den Gletscherdiskurs in den Habsburger Landen, danach während der Bayerischen Besatzungszeit und dann

bereits früher aufgeworfen worden sein. Dieser entscheidende Teil der historischen Klimadiskursforschung bleibt erst noch ein Desiderat.

93 Albrecht von Haller, Alpen (wie Anm. 72), S. 17.

94 Ebd. 
während des Deutschen Bunds und Österreich-Ungarn mitbestimmte. ${ }^{95}$ Seine Beiträge zum Diskurs sind phänomenologischer Natur. Er war ebenso wie de Saussure ein aufgeklärter Naturforscher, der sich fern der Narrative der Alpenbewohner hielt und starke systematisierende Elemente in seiner Phänomenologie aufweist. Für die anthropogenen Einflüsse auf die Gletschervariation und den Klimawandel gibt es bei ihm noch keine Anzeichen. Zu diesen Texten gesellen sich noch eine Reihe weitere im 18. Jahrhundert, die hier unerwähnt blieben. Ein Verweis darf zumindest auf die beiden folgenden Werke nicht fehlen: Johann Georg Altmann mit seinem Werk: „Versuch einer historischen und physischen Beschreibung der helvetischen Eisbergen ".96 Auch der Stuttgarter Gottlieb Conrad Christian Storr beschrieb in seiner "Alpenreise vom Jahre 1781“ ebenfalls die Alpengletscher aus naturforschender Perspektive. ${ }^{97}$

\section{Fazit}

So wichtig das 19. Jahrhundert für die Konstitution der anthropogenen Einflüsse auf das Klima war, so wichtig waren die Jahrhunderte davor für das 19. Jahrhundert gewesen. Es lassen sich drei Phasen feststellen: Ab 1600 schienen die Gletscher, bedingt durch die Kälteperiode ab den 1570er Jahren, die Aufmerksamkeit der Alpenbewohner zu erregen, weil sie Zerstörungen anrichteten. Der Ausbruch des Rofener Eissees 1601 ist ebenso Anzeichen dafür, wie der Untere Grindelwaldgletscher, der zerstörerisch auf St. Petronell und einige Bauernhäuser wirkte. Diese Phase von 1600 bis 1700 ließe sich als eine Intentionalitätsphase des Gletscherdiskurses bezeichnen, in der Zerstörungen für eine erhöhte Aufmerksamkeit sorgten. Dazu gehören die Rebmanns, Merian und auch bis zu einem bestimmten Teil Scheuchzer. Zu einem anderen Teil gehört Scheuchzer bereits in eine andere Phase. Er, Hottinger, dann aber vor allem Martell, Gruner und de Saussure stehen für die Phänomenologiephase. Deskription und Klassifikation von Gletschern unter einem dezidiert naturforschenden Aspekt gaben sie sich zur Aufgabe. Die dritte Phase ist die Analyse- und Explikationsphase aufgrund guter empirischer Grundlagen. Erst im 19. Jahrhundert wurde diese Phase möglich: Schimper, Agassiz, Hugi, Tyndall, de Marchi und Arrhenius stehen mitunter dafür. Durch Schimper und Agassiz verschob sich der Schwerpunkt vom

95 Walcher beeinflusste Zallinger zum Thurn, Johann Georg von Aretin, Joseph Duile und Philipp Krapf.

96 Johann Georg Altmann, Versuch einer historischen und physischen Beschreibung der helvetischen Eisberge, Zürich 1751.

97 Gottlieb Conrad Christian Storr, Alpenreise vom Jahre 1781, Leipzig 1784. 
Gletscherdiskurs zu einem Eiszeitdiskurs. Durch diese Transformation konnte der Jahrhunderte alte Gletscherdiskurs erst in einen Klimawandeldiskurs mutieren, der auch anthropogene Einflüsse nicht übersah, sondern sie dezidiert berücksichtigte. Svante Arrhenius als zentrale Figur für den Klimwandeldiskurs zu begreifen, ist zulässig, weil er mit seinem Aufsatz eine Neuschreibung des Diskurses vorgenommen hat. Der anthropogene Einfluss auf den Klimawandel durch den Treibhauseffekt wird präzise gefasst. Sein genuiner Beitrag zum Klimawandeldiskurs besteht darin, Ernst Lechers Kohlenstoffdioxid-Position mit der von Fourier, de Marchis und Högboms verbunden zu haben. Ernst Lechers Beitrag zum Kohlenstoffdioxid darf dabei nicht unterschätzt werden. Ohne seinen Nachweis, das Kohlenstoffdioxid spiele die entscheidende Rolle für den Treibhauseffekt, hätte Arrhenius nicht den aus unserer Perspektive entscheidenden Schritt gehen können, um seine Diskursposition zu formulieren.

Konsum, Verbrauch und Nutzung von Ressourcen und ihre Auswirkungen auf das Klima würden ohne diese Jahrhunderte des diskursiven Vorlaufs heute wohl nicht so präsent sein. Eine große Chance für das 21. Jahrhundert hätte darin gelegen, wenn der konsumbedingte Klimawandel schon früher in das Bewusstsein der Gesellschaften vorgedrungen wäre. In der Diskursforschung zeigt sich oft die Erkenntnis, dass selbst wenn in Fachdiskursen tiefgreifende Erkenntnisse vorliegen, sie nicht ausreichen, um auch politische Relevanz zu erlangen. Um 1900, die Jahre um das Publikationsjahr von Svante Arrhenius' Aufsatz, gab es wesentlich virulentere Diskurse, als den eines Fouriers, Lechers, Arrhenius' und Högboms. Der Klimawandel konnte offenbar zu einer Zeit, in der Konsum, exothermer Rauch, Qualm und Industrie positiv belegt waren, nicht als ein globales Problem erkannt werden. Insofern ist es nicht verwunderlich, dass es einige Jahrzehnte dauerte, bis es Hermann Flohn Ende der 1970er Jahre gelang, die Weltöffentlichkeit für den Klimawandel durch den anthropogenen Treibhauseffekt zu sensibilisieren. Seitdem sind Klima und Konsum miteinander in der breiten Weltöffentlichkeit verknüpft. Sie provozieren in ihrer Korrelation die Frage nach der zukünftigen Lebensqualität. Eine Antwort darauf, wie zu handeln sei, um Lebensqualität zu erhalten, käme nun spät, aber vielleicht noch nicht zu spät. 